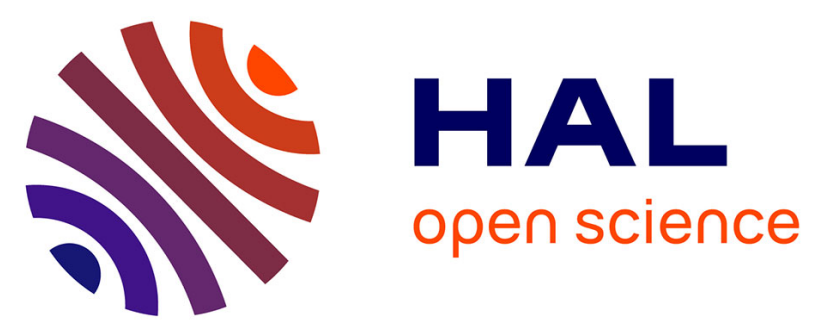

\title{
A class of single and dual-frequency algorithms for rain-rate profiling from a spaceborne radar. Part II: Tests from airborne radar data
}

Paul Amayenc, Jean-Philippe Diguet, Mongi Marzoug, Toufik Tani

\section{- To cite this version:}

Paul Amayenc, Jean-Philippe Diguet, Mongi Marzoug, Toufik Tani. A class of single and dualfrequency algorithms for rain-rate profiling from a spaceborne radar. Part II: Tests from airborne radar data. Journal of Atmospheric and Oceanic Technology, 1996, 13 (1), pp.142-164. 10.1175/15200426(1996)0132.0.CO;2 . hal-00106137

\section{HAL Id: hal-00106137 https://hal.science/hal-00106137}

Submitted on 6 Feb 2021

HAL is a multi-disciplinary open access archive for the deposit and dissemination of scientific research documents, whether they are published or not. The documents may come from teaching and research institutions in France or abroad, or from public or private research centers.
L'archive ouverte pluridisciplinaire HAL, est destinée au dépôt et à la diffusion de documents scientifiques de niveau recherche, publiés ou non, émanant des établissements d'enseignement et de recherche français ou étrangers, des laboratoires publics ou privés. 


\title{
A Class of Single- and Dual-Frequency Algorithmas for Rain-Rate Profiling from Spaceborne Radar. Part II: Tests from Airborme Radar Measurements
}

\author{
Paul Amayenc, Jean Philippe Diguet, * Mongi Marzoug, ${ }^{\dagger}$ and Taoufik Tani \\ Centre d'études des Environnements Terrestre et Planétaires, Issy-les-Moulineaux, France
}

(Manuscript received 22 December 1994, in final form 14 June 1994)

\section{ABSTRACT}

In Part I, four single-frequency (SF) algorithms and a dual-frequency (DF) algorithm for range profiling of the rain rate from a spaceborne radar were described and tested from numerical simulations. In Part II, performances of these algorithms are studied using data from a DF ( $X$ and $K_{\mathrm{a}}$ bands) near-nadir-pointing airborne radar. The data, gathered over ocean near Wallops Island in 1988, mimic future spaceborne radar measurements.

Rain retrievals are performed for isolated and series of contiguous rain measurements within stratiform and convective rain regions overflown by the aircraft. General features and aspects specific to the experiment conditions are pointed out. The SF algorithms provide more or less scattered results according to their own sensitivities to uncorrected scaling errors. Improvement of the algorithms stability by constraining the total pathintegrated attenuation from surface echo measurements is confirmed. The correlation between attenuation coefficients at both frequencies, which forms the theoretical basis of the DF algorithm, is verified from the data. Results from DF algorithm are likely more reliable than SF counterparts since they are globally corrected for scaling errors. Rain-rate thresholds above which "attenuation" algorithms should relay $Z-R$ methods to avoid negative bias in rain-rate estimates are found near $12 \mathrm{~mm} \mathrm{~h}^{-1}$ at X band and $1 \mathrm{~mm} \mathrm{~h}^{-1}$ at $\mathrm{K}_{\mathrm{a}}$ band for a $2.5-\mathrm{km}$ rain depth. Coherent spatial structures of the rain rate within a vertical cross section of the storm are recovered from the "attenuation" algorithms.

The data obtained with a cross-range resolution $L_{0} \approx 1 \mathrm{~km}$ are also used to perform $2 D$ simulations of beamaveraging, and nonuniform beam-filling (NUBF) effects are used for cross-range resolutions $L=2,3$, and 4 $\mathrm{km}$. Degrading the resolution produces a smoothing of small-scale rain-rate structures and a lowering of the rain-rate estimation. Bias due to NUBF depend on the involved "attenuation" algorithm. It increases with $L$ but remains below $15 \%$, up to $L=4 \mathrm{~km}$ for mean rain-rate estimates at large horizontal scale $(\approx 100 \mathrm{~km})$. The $Z$ $R$ methods are weakly affected by the NUBF.

\section{Introduction}

Spaceborne weather radar will play an important role for measuring rainfall from space in the near future (see, e.g., Meneghini and Kozu 1990). Elaborating methods for profiling the rain rate versus range from spaceborne radar measurements led us to define a class of single-frequency (SF) and dual-frequency (DF) algorithms in Part I of the present paper (Marzoug and Amayenc 1994, hereafter referred to as MA94). All the algorithms make use of a common mathematical formalism and perform correction for path-integrated attenuation (or PIA). The potential merit-demerit balance of the proposed algorithms were thoroughly discussed. Results of numerical simulations of algorithms performances using theoretical models of rain-rate pro-

\footnotetext{
* Current affiliation: LASTIENSSAT, Lannion, France.

+ Current affiliation: CNET/PAA, Issy-les-Moulineaux, France.
}

Corresponding author address: Dr. Paul Amayenc, CETP-CNET, 38 rue du General Leclerc, 92131 Issy-les-Moulineaux, France. files were also reported. In this paper, we present application of these algorithms to real data from airborne radar measurements.

Airborne experiments performing sampling of rainy systems from the top are useful to study rainfall retrieval methods. Since some years, an increasing number of airborne radar experiments at frequencies envisioned for future spaceborne measurements were conducted. The data were used in several studies (e.g., Fujita et al. 1985; Meneghini et al. 1989, 1992; Meneghini and Nakamura 1990; Marzoug and Amayenc 1991a, 1992, 1993; Kozu et al. 1991; Amayenc and Marzoug 1992; Iguchi and Meneghini 1994) to test a variety of algorithms envisioned for exploiting future spaceborne radar measurements. Here we used data of such a dedicated experiment.

The present paper rests on the content of Part I (MA94), which is assumed to be known by the reader. The same notations are used. In section 2 , the foundings and the most striking features of the involved algorithms, as deriving from MA94, are briefly recalled. The origin and characteristics of the airborne radar data and the nature of the validation tests used to study the 
algorithms performances are described in section 3 . Then, in section 4, we present results of application of the range-profiling single- and double-frequency (SF and DF, respectively) algorithms to these data and tests of their performances. This is done while ignoring possible nonuniform beam-filling (NUBF) effects that may be a significant source of errors for low-beamresolution measurements usually planned for spaceborne instruments. Simulations of NUBF effects using the airborne radar data are investigated in section 5. Conclusions are given in section 6. Mathematical development dealing with a method to evaluate the PIA over a limited range interval is given in the appendix.

\section{Summary of algorithms characteristics}

The SF algorithms are based upon the use of a $Z$ $=\alpha k^{\beta}$ relation to derive the range-profiled attenuation coefficient further converted into a range-profiled rain rate by means of the $k=c R^{d}$ relationship. Four variants were defined correcting for various types of scaling errors, constant with range, possibly present in radar measurements. The $k Z$ algorithm processing only rain echoes is, in essence, similar to the approach of Hitschfeld and Bordan (1954). It is sensitive to an offset in the radar calibration and to variability in the drop size distribution (DSD) and becomes rapidly unstable when PIA increases. Additional measurements of surface echo attenuation are used as a constraint in two out of four algorithms to correct for scaling errors. The $k Z C$ algorithm corrects for an offset $\delta C$ in the radar calibration but is sensitive to error $\delta A_{r}$ in $\sigma^{0}$ estimate, especially for low PIA, and to DSD variability. It also depends on storm structure along the entire path. The $k Z S$ algorithm corrects for any unknown PIA factor over a range interval $\left(0, r_{0}\right)$ prior to the first processed range gate at range $r_{0}$ by integrating from the surface; thus, it is insensitive to the storm structure at ranges less than $r_{0}$ within the cloud or melting region, for example. It remains, however, sensitive to errors $\delta C$ and $\delta A$, and to DSD variability. The $k Z C$ and $k Z S$ algorithms are generally stable, and the impact of uncorrected error terms decreases with the PIA amount. The fourth algorithm, $k Z N$, assumes constant rain rate versus range within a limited range interval to estimate the attenuation coefficient $k_{m}\left(r_{d}\right)$ at range $r_{d}$ near the surface; then upward integration of "rain signal" is performed to get the range-profile of the attenuation coefficient. It is insensitive to errors $\delta C$ or $\delta A_{t}$ and moderately sensitive to DSD variability. However, the presence of significant rain gradient versus range near $r_{d}$, due to evaporation process, for example, is a possible source of error. Since the four SF algorithms have complementary sensitivities to uncorrected scaling errors, intercomparison of the results was proposed as a means to identify the need for such corrections on individual basis.

The DF algorithm was conceived to improve the correction of scaling error terms possibly present in rain measurements. This is done by exploiting the relationship $k\left(f_{2}\right)=a\left(f_{1}, f_{2}\right) k\left(f_{1}\right)^{b}$ between the attenuation coefficients at two differently attenuating frequencies (with $f_{2}>f_{1}$ ). The nonlinear regression process used to adjust the relation is initialized by means of the various pairs of range-profiled attenuation coefficients provided by the $S F$ algorithms at both frequencies and performs the best fit. Accordingly, the DF algorithm output provides two estimates of the rain-rate profile, one for each frequency. The closeness of these two estimates is a necessary, though not sufficient, condition to assess the credibility of the solution. No explicit use of surface echo measurements is made except in part of the initialization process, implying results of $k Z C$ and $k Z S$ algorithms. The DF approach is more efficient than SF counterparts since it removes scaling errors not corrected in SF algorithms. It also adjusts the DSD-dependent parameters of the rain relations used in the analysis from the initial adjustment of the $a$ parameter in the $k\left(f_{2}\right)-k\left(f_{1}\right)$ relation. Besides, the DF algorithm still works when, for large PIA, the rain signal for the most attenuating frequency $f_{2}$ falls below noise level before the surface range.

\section{Data from airborne radar experiment}

\section{a. Data selection}

During past years, CRL (Communications Research Laboratory, Tokyo) and NASA/GSFC have been conducting a series of joint aircraft experiments using the microwave airborne rain scatterometer/radiometer (MARS) developed at CRL. This "integrated" instrument, originally developed in 1979 (Okamoto et al. 1982) and further improved, includes a downwardlooking DF ( 10.00 and $34.45 \mathrm{GHz}$ ) radar and a DF ( 9.86 and $34.21 \mathrm{GHz}$ ) radiometer. We did not consider measurements in the radiometer mode.

In the 1988-89 experiment, a series of flights with the NASA T-39 Sabreliner jet plane were conducted near Wallops Island, mainly over Chesapeake Bay. The flight altitude, above $10-\mathrm{km}$ altitude, allowed simulation of spacelike observations and examination of heavier convective storms and the upper structure of the precipitation. Most details on the experiment may be found in Kozu (1991), Kozu et al. (1991), and Meneghini et al. (1992). The quality of all datasets was guaranted by eliminating undesirable observations such those corrupted by radio interferences or taken during aircraft attitude fluctuations or banking. We selected the dataset obtained on 1 November 1988 flight as the most interesting one because it included observation of well-organized stratiform and convective rain areas with low to heavy rain. The aircraft flew northeast of Wallops Flight Facility (WWF/NASA) over the ocean between 1945 and 2230 UTC. At WFF, the altitude of $0^{\circ} \mathrm{C}$ isotherm was about $3 \mathrm{~km}$, and the surface temperature was close to $11^{\circ} \mathrm{C}$. 
TABLE 1. Major system parameters for the dual-frequency radar onboard T-39 aircraft (adapted from Kozu 1991).

\begin{tabular}{lcc}
\hline \multicolumn{1}{c}{ Parameter } & X band & $\mathrm{K}_{\mathrm{a}}$ band \\
\hline Center frequency & $10.00 \mathrm{GHz}$ & $34.45 \mathrm{GHz}$ \\
Antenna & & \\
Type & Horn lens & Horn lens \\
Polarization & Linear VV & Linear VV \\
Aperture & $42 \mathrm{~cm}$ & $13 \mathrm{~cm}$ \\
Gain & $30.3 \mathrm{~dB}$ & $30.4 \mathrm{~dB}$ \\
Half-power beamwidth & $5.2^{\circ}$ & $5.2^{\circ}$ \\
Scanning angle & Approx. nadir & Approx. nadir \\
& pointing & pointing \\
Transmitter & & \\
Peak power & $20 \mathrm{~kW}$ & $10 \mathrm{~kW}$ \\
PRF & $440 \mathrm{~Hz}$ & $440 \mathrm{~Hz}$ \\
Pulse width & $0.5 \mu \mathrm{s}$ & $0.5 \mu \mathrm{s}$ \\
Receiver & & \\
Noise figure & $5.3 \mathrm{~dB}$ & $9.6 \mathrm{~dB}$ \\
Detection & Logarithmic & Logarithmic \\
Dynamic range & $80 \mathrm{~dB}$ & $80 \mathrm{~dB}$ \\
Signal processor & & \\
Sample interval & $0.2 \mu \mathrm{s}$ & $0.2 \mu \mathrm{s}$ \\
Range window & $22.5 \mathrm{~km}$ & $20.25 \mathrm{~km}$ \\
No. of independent & 128 & 128 \\
samples (nominal) & & \\
\hline
\end{tabular}

\section{b. Experiment conditions and data processing}

The main system parameters for the dual-frequency radar are listed in Table 1 . The nominal cruising altitude of the aircraft was $11-12 \mathrm{~km}$, with the $X$ - and $\mathrm{K}_{\mathrm{a}}$ band antennas maintained fixed at near-nadir incidence. In fact, the mean incidence was generally very close to $5^{\circ}$ off nadir, due to aircraft attitude. Thus, the rangegated measurements combined with aircraft motion yielded almost vertical cross section of the overflown precipitating systems.

The nearly identical half-power beamwidths of about $5^{\circ}$ provide a $1-\mathrm{km}$ surface footprint and well-matched observation volumes within precipitation at both frequencies. Note that the 3-dB footprint actually corresponds to 6-dB beamwidth because the antenna gain function is applied for both transmission and reception. The small footprint size allows us to consider that NUBF effects are almost negligible. The nominal cruising speed of the aircraft is about $200 \mathrm{~m} \mathrm{~s}^{-1}$. Each radar observation for an entire path, enabling observation of rain, surface, and mirror echoes within the range window, consists of a 128-sample average over approximately $0.4 \mathrm{~s}$, which corresponds to a horizontal displacement of the aircraft of about $80 \mathrm{~m}$. With $1-\mathrm{km}$ footprint, there is a large overlap of radar beams from one observation to the next one. Independent measurements for contiguous footprints are actually obtained every $4.8 \mathrm{~s}$, or every 12 observations. One minute of data, or 150 observations, corresponds to an "equivalent" horizontal length of about $12 \mathrm{~km}$ if observation is continuous.

Special care was taken by the experimenters to perform accurate radar calibration. The method included internal calibration using calibrated signal and noise sources, external calibration process using radar measurements at $X$ band for an over-rain gauge flight above land, and adjustments of radar return powers at $\mathrm{X}$ and $\mathrm{K}_{\mathrm{a}}$ bands from small ice scatterers above the bright band during light stratiform rain. Details and results may be found in Kozu (1991) and Kozu et al. (1991).

The mean returned power measurements within rain were first corrected by subtracting the mean noise power estimated within "no rain" gates along the path, then converted into reflectivity factors by means of the calibration procedure provided by the experimenters. The pulse duration of $0.5 \mu$ s at each frequency provided a nominal range resolution of $75 \mathrm{~m}$. The return pulse, however, was oversampled at a 5-MHz rate, that is, a $0.2-\mu$ s interval or $30 \mathrm{~m}$. Thus, reflectivities data were generated over contiguous $75-\mathrm{m}$ gated intervals by us-
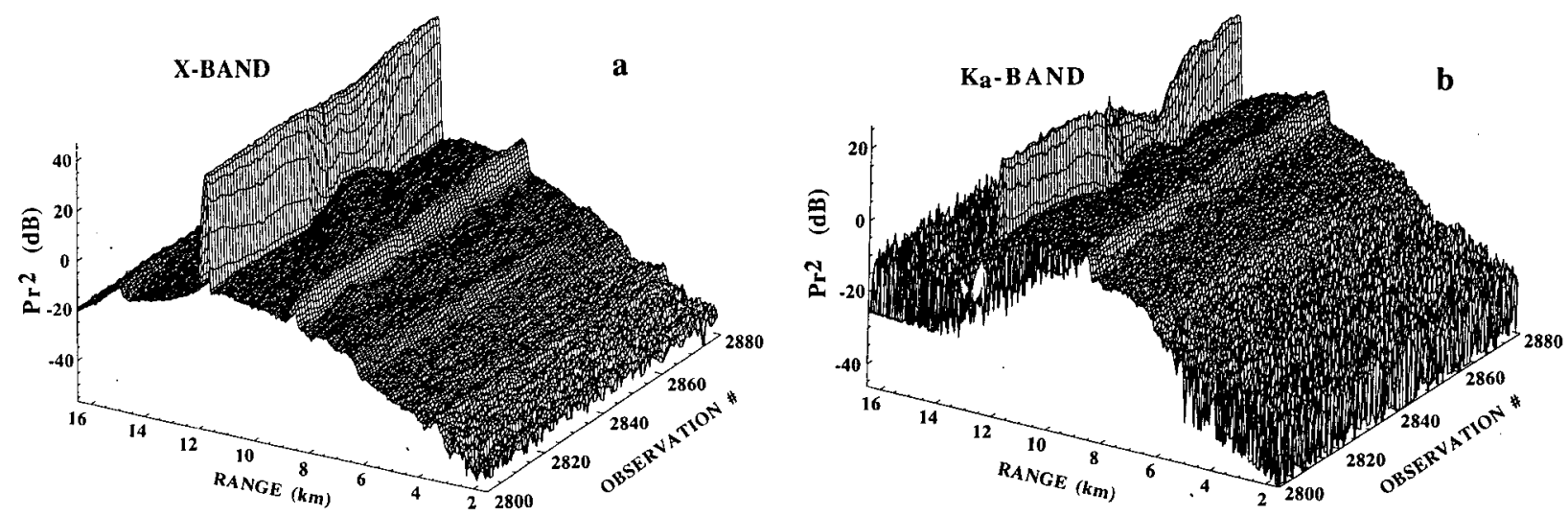

FIG. 1. Example of 3D plot of selected profiles of range-normalized mean received power $\operatorname{Pr}^{2}$ (in arbitrary decibels) in the $1.5-16.5-\mathrm{km}$ range interval (a) at $\mathrm{X}$ band and (b) at $\mathrm{K}_{\mathrm{a}}$ band for the 1 November 1988 experiment over ocean. The sequential observation number ranges from 2800 to 2880 . 
ing weighted running means of the genuine data. The minimum detectable "apparent" reflectivity factor next to the surface (11-km range) was about $15 \mathrm{dBZ}$ at both frequencies.

Figure 1 shows an example of $3 \mathrm{D}$ plots of the mean received power profile normalized to squared range $r^{2}$ for an ensemble of 81 contiguous observations (see Fig. 3) at $X$ band and $K_{a}$ band in the $1.5-16.5-\mathrm{km}$ range interval. The large peak at about $11.2-\mathrm{km}$ range is the surface return. At X band, the surface return is almost constant indicating low PIA. At $\mathrm{K}_{\mathrm{a}}$ band, larger PIA is evident from variations in the surface return. The $\mathrm{K}_{\mathrm{a}}$-band signal is largely contaminated by noise near aircraft and deep in the rain. Below the surface, the mirror image return is present at $\mathrm{X}$ band, while it disappears at $K_{a}$ band. A brightband, less pronounced at $\mathrm{K}_{\mathrm{a}}$ band than at $\mathrm{X}$ band, is present near range $8.2 \mathrm{~km}$. Figure 2 shows a 3D plot of the difference between "apparent" rain reflectivities factors at both frequencies: $\Delta Z=Z_{m, \mathrm{X}}-Z_{m, \mathrm{~K}}$. ${ }^{\text {'T }}$ The plot is limited to the range interval $5-11 \mathrm{~km}$. From low to large ranges, $\Delta Z$ shows first a chaotic region dominated by noise effects, then a peak near range $8.2 \mathrm{~km}$ due to difference in the brightband magnitudes at both frequencies, and finally a monotonic increase due to growing differential pathattenuation.

The surface echo power measurements were processed to get "apparent" values $\sigma_{m}^{0}$ of the surface backscatter coefficient below rain. At $X$ band, the surface echo was always found detectable, even for heavy rain, and $\sigma_{m}^{0}$ was computed from the observed peak value of the surface return. At $\mathrm{K}_{\mathrm{a}}$ band, $\sigma_{m}^{0}$ was determined in the same manner when surface return was detected. For heavy rain, however, when the surface return was masked by noise, $\sigma_{m}^{0}$ was computed using the noise level at the surface range. Obviously, this underestimated the total PIA in the rain layer. For each frequency, the reference value of $\sigma^{0}$ outside rainy areas was also determined (see section $4 a$ ).

\section{c. Nature of the validation tests}

As most experiments with T-39 in 1988-89, the selected experiment did not involve permanent means, such as rain gauge networks or ground-based radars, enabling "external" validation of the rain structures retrieved from the various algorithms. This came mainly from the fact that the overflown rainy areas were most frequently located over ocean, too far from the ground-based SPANDAR radar in operation at WFF or from existing land-based rain gauges. Thus, we had to conceive "internal" procedures to validate results of aircraft radar. The basic step was to compare rain profiles recovered from every algorithm at $\mathrm{X}$ band

\footnotetext{
' In the following, subscripts $\mathrm{X}$ and $\mathrm{K}$ stand for $\mathrm{X}$ band and $\mathrm{K}_{\mathrm{a}}$ band, respectively.
}

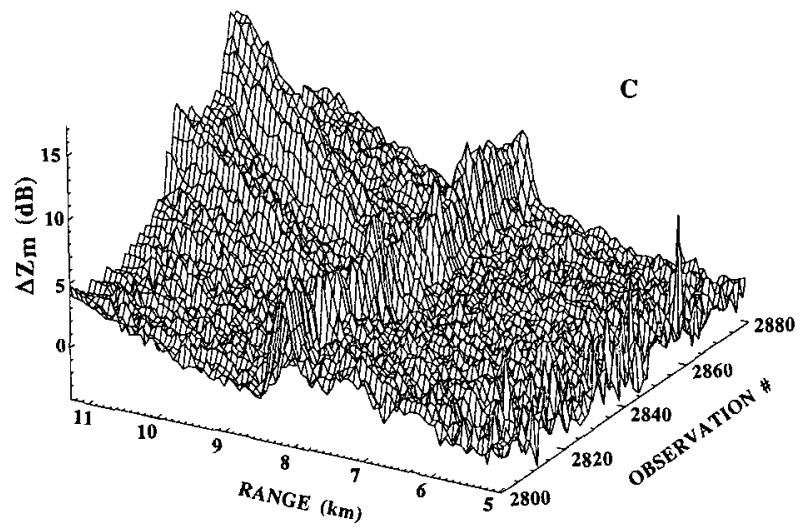

FIG. 2. Example of 3D plot of the difference $\Delta Z_{m}=Z_{m . X}-Z_{m, \mathrm{~K}}$ (dB) between the apparent rain reflectivity factors at both frequencies. The sequential observations ranging from 2800 to 2880 are the same as in Fig. 1.

and $K_{a}$ band, since the involved PIA effects corrected by the algorithms are different (cf. Table 3 ). These comparisons were studied for selected profiles or for series of contiguous profiles. In the latter case, we used mean rain rates over the rain-layer depth, derived from profiles retrieved from various algorithms. We also analyzed reconstructed $2 \mathrm{D}$ rain structures within the vertical alongtrack plane. Besides, interpretation of the experimental results profited by significant inputs from theoretical simulation results (e.g., Marzoug and Amayenc 1991b; MA94).

\section{Application of SF and DF algorithms: Results and discussion}

\section{a. Algorithms processing}

Some specific aspects of the algorithms processing rounding off the general description given in MA94 and the elements given in section 2 are hereafter made clear.

To process $k Z C$ and $k Z S$ algorithms, the model of $\sigma^{0}$ outside rain was determined by processing surface returns in clear areas as close as possible to the raining areas observed during the flight. Data were first ranked according to the beam incidence, within angular intervals of $2^{\circ}$ centered from $3^{\circ}$ to $9^{\circ}$, then averaged within each angular interval. For the most frequent incidence, near $5^{\circ}$, involving about 1150 data points, the mean value was $\left\langle\sigma^{0}\right\rangle \approx 1.8 \mathrm{~dB}(4.2 \mathrm{~dB})$, with a standard deviation of $0.5 \mathrm{~dB}(1.4 \mathrm{~dB})$ at $\mathrm{X}$ band $\left(\mathrm{K}_{\mathrm{a}}\right.$ band).

For cases where rain signal was obscured by noise in the range interval $\left(r_{d}, r_{s}\right)$, as commonly observed for large PIA at $\mathrm{K}_{\mathrm{a}}$ band, a procedure assuming constant rain rate in this interval to correct for the unknown PIA factor $A\left(r_{d}, r_{s}\right)$ was included in $k Z C$ and $k Z S$ algorithms (MA94).

The slope technique, used in the kZN algorithm to determine $k_{m}\left(r_{d}\right)$, was applied over four contiguous 


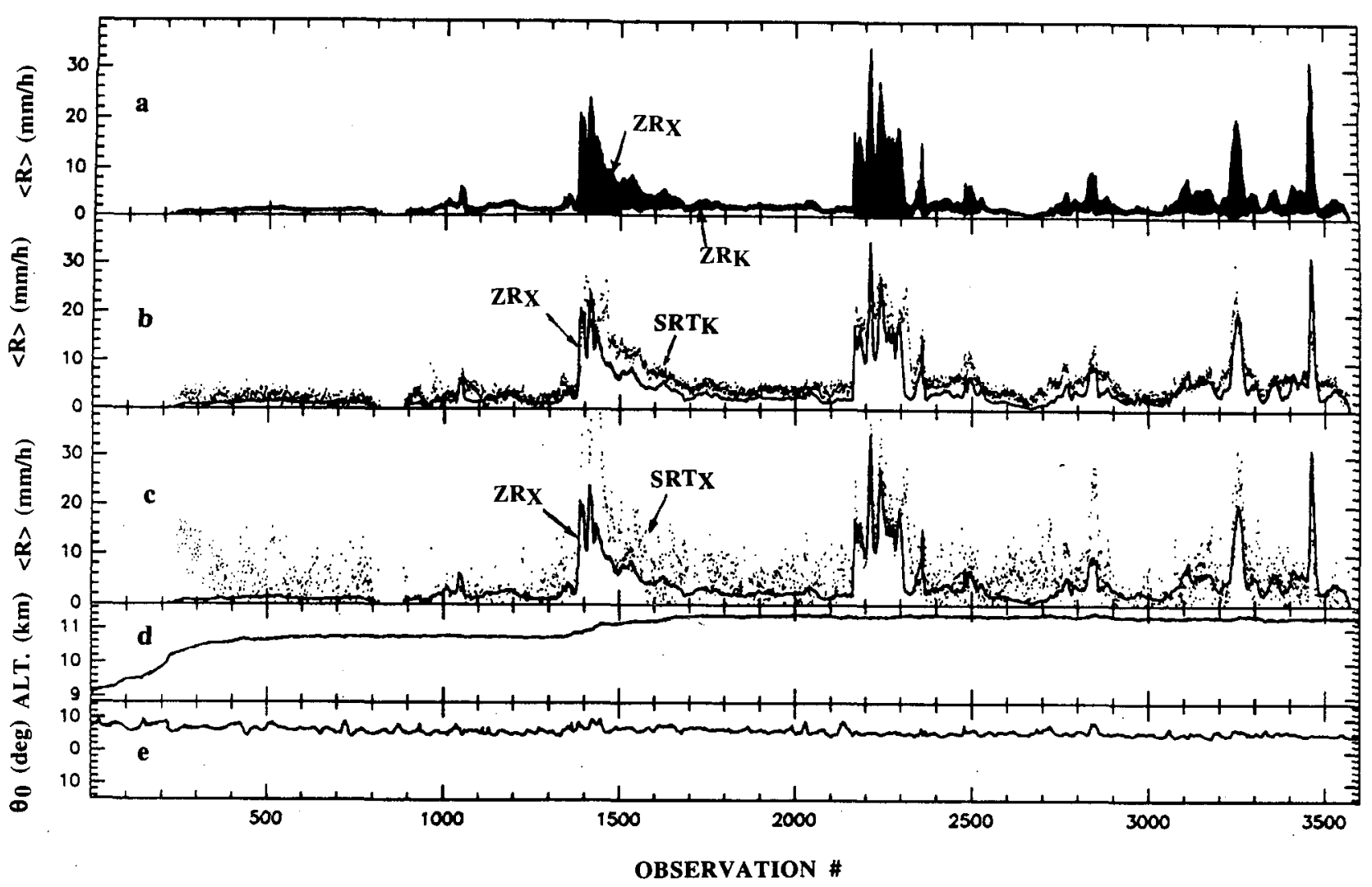

FIG. 3. Display of several parameters for increasing observation numbers derived from the dataset on 1 November 1988. The panels show (a) the mean (layer-averaged) rain rates $\left\langle R_{\mathrm{X}}\right\rangle\left(\left\langle R_{\mathrm{K}}\right\rangle\right)$ computed from the backscatter method at $\mathrm{X}$ band $\left(\mathrm{K}_{\mathrm{a}}\right.$ band) using the $Z R_{\mathrm{X}}\left(Z R_{\mathrm{K}}\right)$ relationship (the dashed area points out difference between the two estimates); (b) the mean (layer-averaged) rain rate from $S R T_{K}$ (dots) compared with $\left\langle R_{\mathrm{X}}\right\rangle$ (full line); (c) the mean (layer-averaged) rain rate from SRT (dots) compared with $\left\langle R_{\mathrm{X}}\right\rangle$ (full line); (d) the aircraft flight altitude above MSL; (e) the off-nadir incidence of the radar beam. Note that 100 contiguous observations (gathered during about $40 \mathrm{~s}$ ) are equivalent to a horizontal distance of about $8 \mathrm{~km}$.

gates where $\mathrm{SNR}>3 \mathrm{~dB}$ near range $r_{d}$, the closest as possible to the surface range $r_{s}$.

For DF algorithm, an automatic procedure was used to identify the $r_{1}-r_{2}$ range interval where difference in rain reflectivities at both frequencies increased monotonically versus range, enabling exploitation of the differential path-attenuation effect (see Fig. 2). Possible difference in the brightband region, where differences in reflectivity may result from non-Rayleigh scattering rather than differential path attenuation, was excluded.

TABLE 2. Relations between the integrated rainfall parameters [with $Z$ in its usual units $\left(\mathrm{mm}^{6} \mathrm{~m}^{-3}\right), k$ in decibels per kilometer, and $R$ in millimeters per hour] used in the algorithms to process the airborne radar data at $\mathrm{X}$ band and $\mathrm{K}_{\mathrm{a}}$ band.

\begin{tabular}{lll}
\hline \hline Relation & \multicolumn{1}{c}{$\mathrm{X}$ band $(10 \mathrm{GHz})$} & $\mathrm{K}_{\mathrm{a}}$ band $(35 \mathrm{GHz})$ \\
\hline$Z-R$ & $Z_{\mathrm{X}}=204 \mathrm{R}^{1.6}$ & $Z_{\mathrm{K}}=314 \mathrm{R}^{1.3}$ \\
$k-R$ & $k_{\mathrm{X}}=0.014 \mathrm{R}^{1.136}$ & $k_{\mathrm{K}}=0.219 \mathrm{R}^{1.047}$ \\
$Z-k$ & $Z_{\mathrm{X}}=8.315 \times 10^{4} k_{\mathrm{X}}^{1.408}$ & $Z_{\mathrm{K}}=2.09 \times 10^{3} k_{\mathrm{K}}^{1.247}$ \\
$k-k$ & & $k_{\mathrm{K}}=11.6 \mathrm{k}_{\mathrm{X}}^{0.92}$ \\
\hline
\end{tabular}

The $r_{1}-r_{2}$ interval was required to include at least five contiguous range gates, with detectable rain signals ( $\mathrm{SNR} \geqslant 3 \mathrm{~dB}$ ) at both frequencies.

The relations between the integrated rain parameters $Z, k$, and $R$, used to run the algorithms, are listed in Table 2. They are similar to those used in previous studies (e.g., Kozu 1991; Kozu et al. 1991; Meneghini et al. 1992) dealing with tests of rain retrieval algorithms from similar data. The $Z-R$ and $k-R$ relations are taken from Atlas and Ulbrich (1977), who used Mie calculation and power-law fits to a set of measured drop size distributions, except the $Z_{\mathrm{X}}-R$ law based on the DSD model of Marshall and Palmer (1948). The $Z_{X, \mathrm{~K}}-k$ relations used in SF algorithms, and the $k_{\mathrm{K}}-k_{\mathrm{X}}$ relation used in DF algorithm, are derived from them.

It is helpful to indicate the expected PIA as a function of rain rate and frequency. This is given in Table 3 using $k-R$ laws of Table 2 with constant rain rate $\langle R\rangle$ over a 3-km rain depth. For completeness, a PIA of $3 \mathrm{~dB}(10 \mathrm{~dB})$ is produced by $\left\langle R_{\mathrm{x}}\right\rangle=23.3 \mathrm{~mm} \mathrm{~h}^{-1}$ $\left(67.3 \mathrm{~mm} \mathrm{~h}^{-1}\right)$ and $\left\langle R_{\mathrm{K}}\right\rangle=2.2 \mathrm{~mm} \mathrm{~h}^{-1}\left(7 \mathrm{~mm} \mathrm{~h}^{-1}\right)$. Note that the PIA exceeds the observed standard de- 
TABLE 3. Expected values of the total two-way PIA at $X$ band $\left(P I A_{X}\right)$ and $K_{a}$ band $\left(P I A_{K}\right.$ ) over a $3-\mathrm{km}$-deep rain layer with uniform rain rate $\langle R\rangle$ using $k-R$ relations in Table 2 .

\begin{tabular}{lcccccc}
\hline$\langle R\rangle\left(\mathrm{mm} \mathrm{h}^{-1}\right)$ & 1 & 2 & 5 & 10 & 20 & 40 \\
\hline PIA $_{\mathrm{X}}(\mathrm{dB})$ & 0.08 & 0.18 & 0.53 & 1.15 & 2.52 & 5.55 \\
PIA $_{\mathrm{K}}(\mathrm{dB})$ & 1.31 & 2.72 & 7.09 & 14.6 & 30.3 & 62.5 \\
\hline
\end{tabular}

viation in $\sigma^{0}$ estimate for $\left\langle R_{\mathrm{X}}\right\rangle$ above $6 \mathrm{~mm} \mathrm{~h}^{-1}$ and $\left\langle R_{\mathrm{K}}\right\rangle$ above $1.1 \mathrm{~mm} \mathrm{~h}^{-1}$. For rain rates below these values, uncertainties in $\sigma^{0}$ estimates may have significant impact on the accuracy of path-averaged rain rates deduced from the surface reference technique (or SRT), as shown by Meneghini et al. (1992).

\section{b. Overview of attenuation effects}

The selected dataset includes 3600 observations. Several parameters characterizing these observations are displayed in Fig. 3. The aircraft altitude flight, shown in Fig. 3d, slowly varies from 9.2 to $11.4 \mathrm{~km}$. The off-nadir incidence of the radar beam, shown in Fig. 3e, ranges between $4^{\circ}$ and $10^{\circ}$, and most frequently in the $5^{\circ}-6^{\circ}$ angular interval. Estimates of the mean layer-averaged rain rate $\left\langle R_{\mathrm{X}}\right\rangle$ and $\left\langle R_{\mathrm{K}}\right\rangle$ are shown in Fig. 3a. They were obtained by using $Z_{\mathrm{X}}-R$ and $Z_{\mathrm{K}}-$ $R$ relations (cf. Table 2) to get $R$ profile from apparent reflectivity $Z_{m}$ above a $15-\mathrm{dB} Z$ threshold, then averaging versus height. At $X$ band, where PIA effects remain moderate, $\left\langle R_{\mathrm{X}}\right\rangle$ points out rather well the mean rain-rate structure. Several zones of heavy rain are observed near observations $1400,2200,3250$, and 3450 . Though possibly affected by uncorrected PIA effects for moderate to heavy rain, the $\left\langle\boldsymbol{R}_{\mathrm{X}}\right\rangle$ estimate may be taken as a reference for comparison to characterize stronger attenuation effects at $\mathrm{K}_{\mathrm{a}}$ band. Here $\left\langle R_{\mathrm{K}}\right\rangle$ agrees with $\left\langle R_{\mathrm{X}}\right\rangle$ for low rain rates only $\left(\leqslant 2 \mathrm{~mm} \mathrm{~h}^{-1}\right.$, typically). Above this threshold, attenuation at $\mathrm{K}_{\mathrm{a}}$ band produces increasingly negative bias in $\left\langle R_{\mathrm{K}}\right\rangle$, and "saturation" effect occurs. Meneghini et al. (1989) gave theoretical estimation of the saturation threshold in $\left\langle R_{\mathrm{K}}\right\rangle$ estimate using a uniform rain model, in agreement with the observations. The path-averaged rain rate estimated from surface echo attenuation using $\mathrm{SRT}_{K}$ $\left(\mathrm{SRT}_{\mathrm{X}}\right)$ is compared with $\left\langle R_{\mathrm{X}}\right\rangle$ in Fig. 3b (Fig. 3c). $\mathrm{The} \mathrm{SRT}_{\mathrm{K}}$ estimate, which is not too affected by uncertainties in $\sigma^{0}$ estimates owing to large PIA at $\mathrm{K}_{\mathrm{a}}$ band, is in fair agreement with $\left\langle R_{\mathrm{X}}\right\rangle$. Conversely, large scatter and strong deviations from $\left\langle R_{\mathrm{X}}\right\rangle$ are observed in SRT $\mathrm{T}_{\mathrm{x}}$ estimate. This occurs when the PIA and the mean error in $\sigma^{0}$ are of the same order of magnitude (cf. Table 3): the $\mathrm{SRT}_{\mathrm{X}}$ may even produce meaningless "negative"' rain-rate estimates (not plotted in Fig. 3c). Meneghini et al. $(1987,1992)$ have shown that a dualfrequency version of the SRT improved the path-averaged rain estimates by reducing the impact of uncertainties in $\sigma^{0}$.
We searched for an experimental verification of the $k_{\mathrm{K}}-k_{\mathrm{X}}$ relation between attenuations at both frequencies, which constitutes the theoretical basis of the DF algorithm. For this purpose, we used estimates of the two-way PIA factor $A\left(r, r_{s}\right)$ at $\mathrm{X}$ and $\mathrm{K}_{\mathrm{a}}$ bands derived from MA94, and we made this clear in the appendix. Neglecting uncorrected error terms in (A4) yields

$$
\begin{aligned}
A\left(r, r_{s}\right)=[1 & +\left(\frac{0.46}{\beta}\right) \\
& \left.\times\left(A_{t m} \alpha\right)^{-1 / \beta} \int_{r}^{r_{s}} Z_{a m}^{1 / \beta}(r) d s\right]^{-\beta},
\end{aligned}
$$

where $A_{t m}$ is the "measured" surface echo two-way attenuation factor, $Z_{m}(r)$ is the apparent rain reflectivity at range $r$, and $r_{s}$ is the surface range.

The PIA factors were calculated over the range interval $\left(r, r_{s}\right)$ covering the first $2 \mathrm{~km}$ above the surface, and then used to determine the correlation between the mean attenuation coefficients at both frequencies. The results are shown in Fig. 4. The "experimental" power-law best fit deriving from 2801 observations (i.e., 800 to 3600 ) is

$$
k_{\mathrm{K}}=10.54 k_{\mathrm{X}}^{0.775}
$$
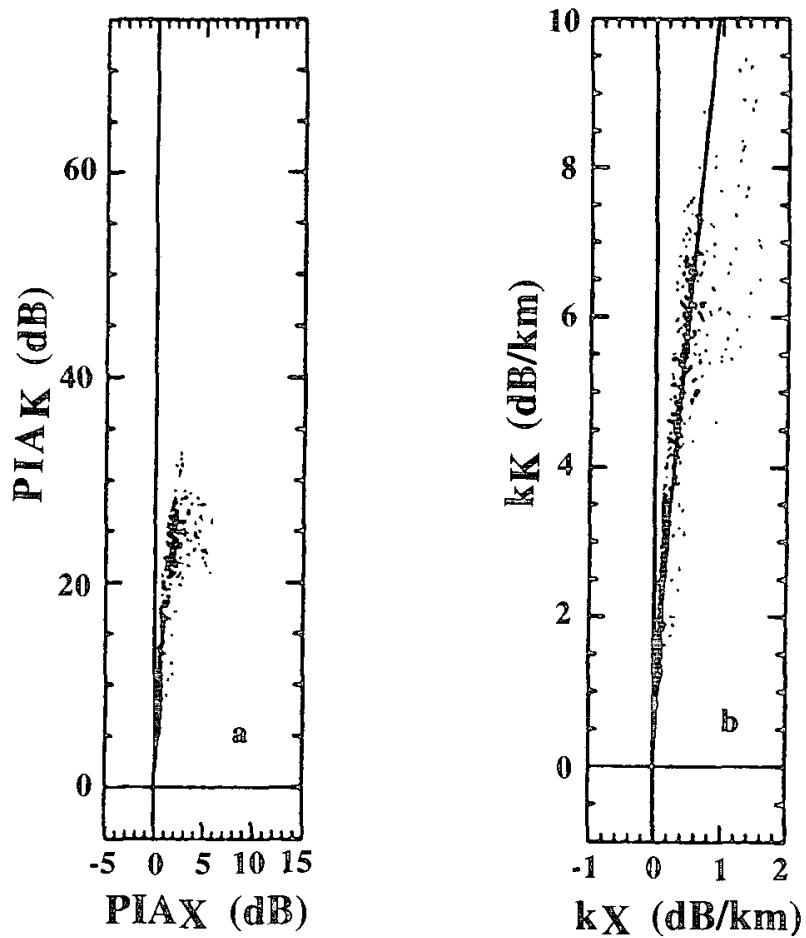

Fig. 4. Overall attenuation effects computed from 2801 data pairs (observations 800-3600 on 1 November 1988): (a) scatterplot of the two-way PIA at $K_{a}$ band versus $X$ band integrated over the first $2 \mathrm{~km}$ (above the surface) of the rain layer; (b) corresponding mean specific attenuation coefficients $\left(\mathrm{dB} \mathrm{km}^{-1}\right)$ at $K_{\mathrm{a}}$ band versus $\mathrm{X}$ band and best power-law fit to the results. 

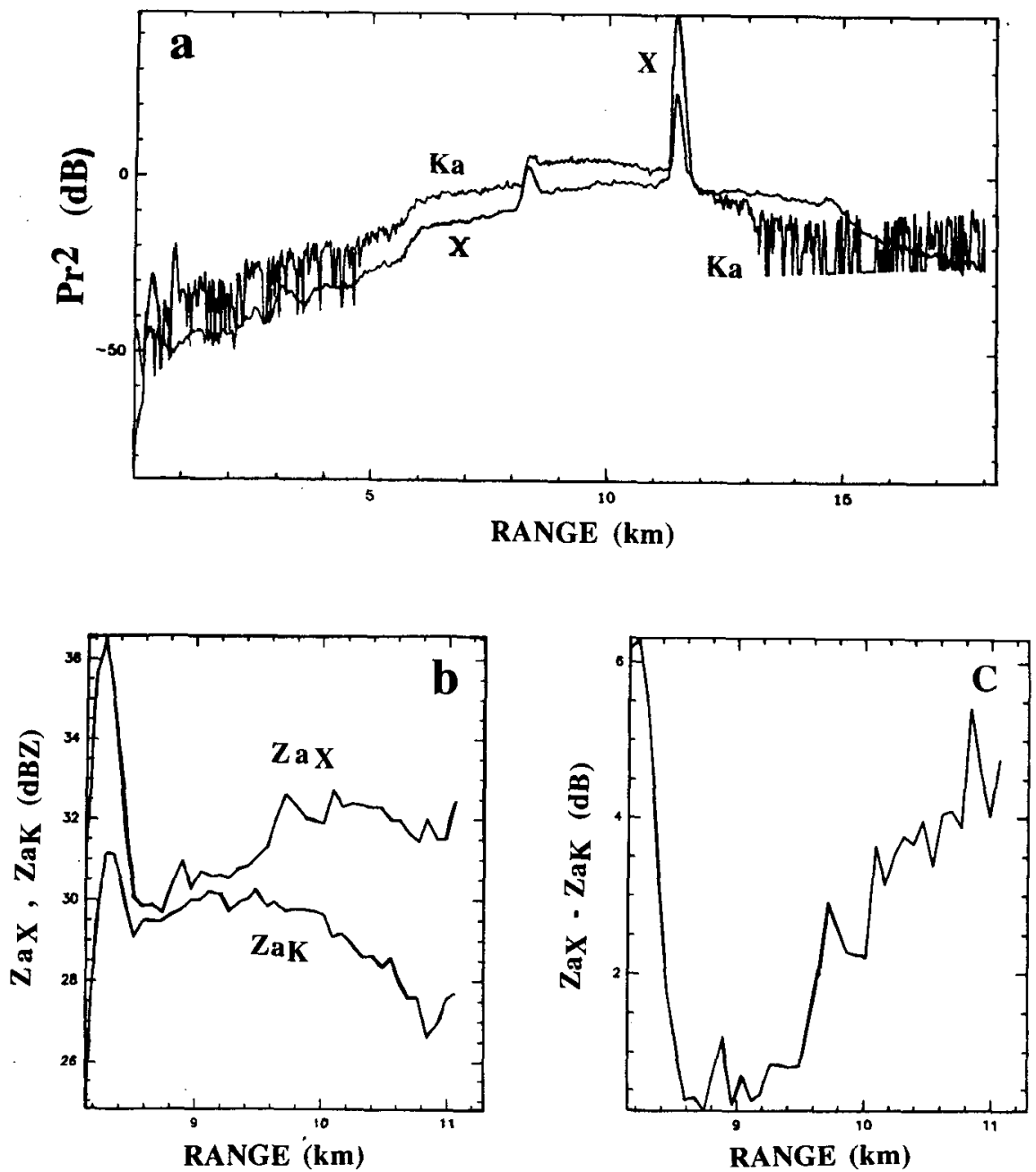

FiG. 5. Example of radar measurements within stratiform rain (observation 2821, off-nadir beam incidence $6.1^{\circ}$ ). Range-profiles of (a) range-normalized received powers $\mathrm{Pr}^{2}$, (b) apparent reflectivities $Z_{\mathrm{X}}$ and $Z_{\mathrm{K}}$, and (c) the difference in apparent reflectivity $\left(Z_{\mathrm{X}}-Z_{\mathrm{K}}\right)$ at $X$ and $\mathrm{K}_{\mathrm{a}}$ bands.

with a high value of the correlation coefficient, $\rho$ $=0.996$.

This "experimental" law compares rather well with the selected "empirical" law (see Table 2):

$$
k_{\mathrm{K}}=11.2 k_{\mathrm{X}}^{0.92} .
$$

When total PIA provided by measurements of surface echo attenuation are used for the same purpose, the obtained experimental law, $k_{\mathrm{K}}=5.40 k_{\mathrm{X}}^{0.545}$ with $\rho$ $=0.712$, worsens. This comes from uncertainties in the PIA (see Fig. 3) for low rain rates that are also responsible for occasional nonphysical "negative" values. Conversely, (1) always yields positive PIA estimates, though it is still sensitive to errors in $\sigma^{0}$ (see the appendix). We also verified that (2) was not too much affected by uncertainties in $\alpha$ coefficient of the $Z=\alpha k^{\beta}$ relation at each frequency. The obtained $a(b)$ parameter in the $k_{\mathrm{K}}=a k_{\mathrm{X}}^{b}$ relation varied from 9.1 to 11.5
( 0.72 to 0.92 ) for $\pm 50 \%$ changes in $\alpha$, while $\rho$ $(\geqslant 0.96)$ remained very high.

For running the DF algorithm, however, we used (3) (cf. Table 2) instead of the "experimental" law (2) in order to preserve a full coherency between all relations involved in the analysis. Let us be reminded, however, that the parameter $a$ is adjusted in the DF algorithm processing (MA94).

\section{c. Selected results for isolated rain profile retrievals}

The study is now focused on the data subset covering observations $2000-3600$, which includes low to heavy rain-rate cases. The 1601 observations in question correspond to an acquisition time of $640 \mathrm{~s}$, or about 128 $\mathrm{km}$ along track, within the rainy system. Two typical examples of the isolated rain-rate profile retrieved from SF and DF algorithms for stratiform and convective precipitation are presented below. 

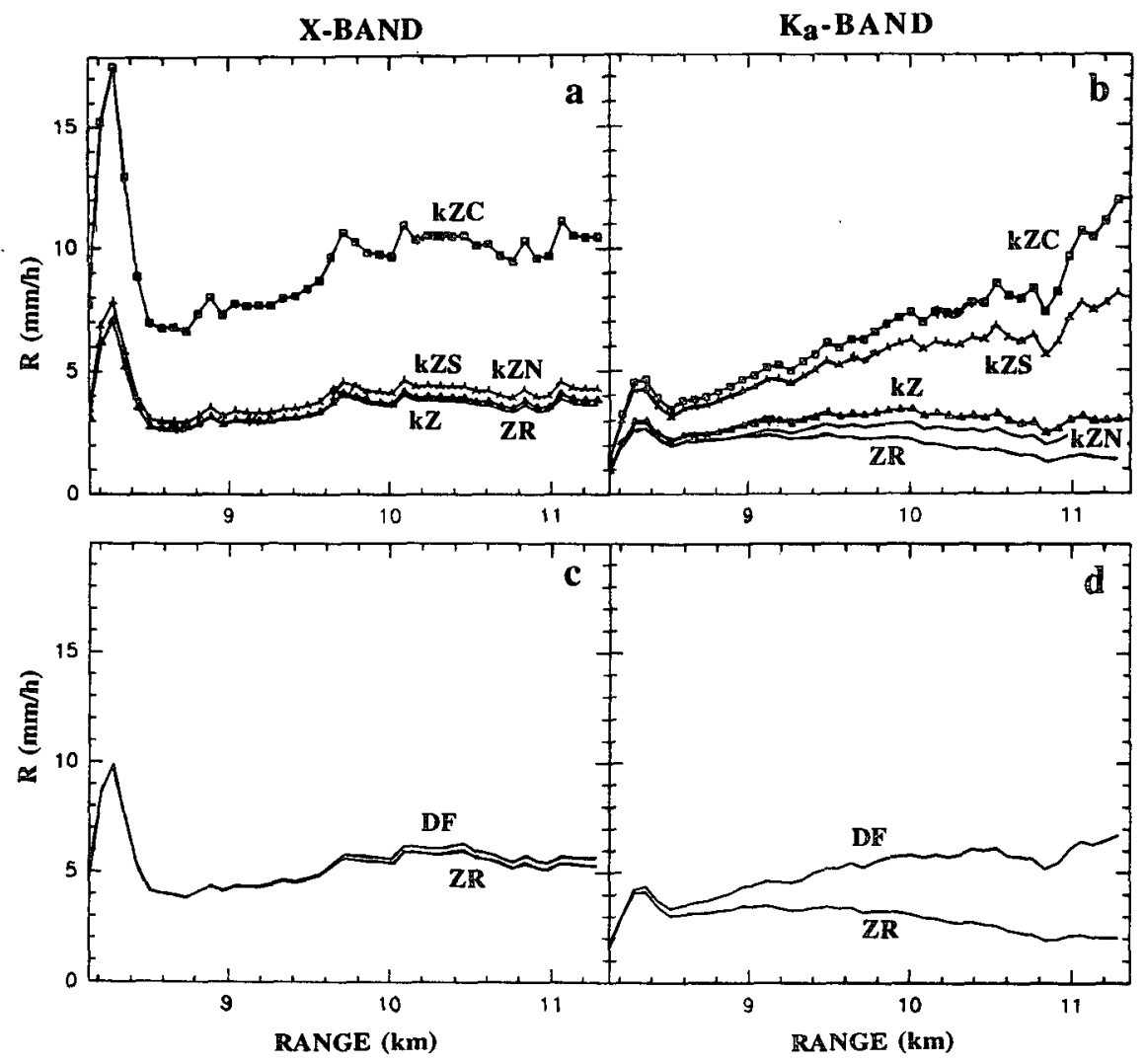

FiG. 6. Example of range-profiled rain rates within stratiform rain (observation 2821, off-nadir beam incidence $6.1^{\circ}$ ) retrieved from the SF algorithms and the $Z-R$ algorithm (a) at $\mathrm{X}$ band and (b) at $\mathrm{K}_{\mathrm{a}}$ band, and from the DF algorithm and the "corrected" $Z^{\mathrm{cor}}-R$ algorithm (c) at $\mathrm{X}$ band and (d) at $K_{\mathrm{a}}$ band. See radar measurements in Fig. 5.

The first example corresponds to observation 2821 (see Fig. 3) in stratiform rain with low rain rate. In Fig. 5 the range-normalized rain powers are plotted, along with the derived apparent reflectivities at both frequencies and their difference $\Delta Z=Z_{\mathrm{X}}-Z_{\mathrm{K}}$ over the last $3-\mathrm{km}$ range interval. The brightband peak, more pronounced at $\mathrm{X}$ band than at $\mathrm{K}_{\mathrm{a}}$ band, is seen near range $8.3 \mathrm{~km}$. In Fig. $5 \mathrm{a}$, the peak is also seen in mirror image at $X$ band near range $14.6 \mathrm{~km}$, while signal at $K_{a}$ band is obscured by noise beyond range $13 \mathrm{~km}$. Below the bright band, $\Delta Z$ increases monotonically from $0 \mathrm{~dB}$ near range $8.6 \mathrm{~km}$ to $4 \mathrm{~dB}$ near the surface, while ignoring Rayleigh fluctuations.

In Fig. 6, the upper plots show the results for SF and $Z-R$ algorithms. At $\mathrm{X}$ band, where $\mathrm{PIA} \mathrm{A}_{\mathrm{X}}=1.2 \mathrm{~dB}$, the $k Z-, k Z S$-, and $k Z N$ algorithm results are almost identical and close to the $Z-R$ estimate $\left(\langle R\rangle \approx 4 \mathrm{~mm} \mathrm{~h}^{-1}\right.$ below the bright band). Here $k Z C_{\mathrm{X}}$ yields high value $\left(\langle R\rangle \approx 10 \mathrm{~mm} \mathrm{~h}^{-1}\right)$ likely due to high sensitivity to uncertainty in PIA estimate for such a low rain rate. At $\mathrm{K}_{\mathrm{a}}$ band, where PIA $\mathrm{K}_{\mathrm{K}}=10.2 \mathrm{~dB}$, the $Z-R$ algorithm obviously underestimates the rain rate. Here $k Z_{K}$ and $k Z N_{\mathrm{K}}$, which do not use surface echo, produce almost similar low-attenuation correction, and their results $\left(\langle R\rangle \approx 3 \mathrm{~mm} \mathrm{~h}^{-1}\right)$ are close to those obtained at $\mathrm{X}$ band; $k Z C_{\mathrm{K}}$ and $k Z S_{\mathrm{K}}$, which use surface echo, both overestimate the attenuation correction. It was verified that a lowering of $0.7 \mathrm{~dB}$ in PIA $\mathrm{X}_{\mathrm{X}}$ and of $1.9 \mathrm{~dB}$ in PIA $_{K}$, slightly in excess of $\sigma^{0}$ uncertainties, could put results of $k Z C_{\mathrm{X}}, k Z C_{\mathrm{K}}$, and $k Z S_{\mathrm{K}}$ in good accordance with those of other "attenuation" algorithms.

In Fig. 6 , the lower plots show the two $R$ profiles provided by the DF algorithm at $X$ band and at $K_{a}$ band, respectively, along with the $Z-R$ estimate for each case. Here, however, the plotted $Z_{\mathrm{X}}^{\mathrm{cor}}-R$, and $Z^{\mathrm{cor}}-R$ estimates ${ }^{2}$ were corrected for "calibration errors" in $Z_{\mathrm{X}}$ or $Z_{\mathrm{K}}$ derived from the DF algorithm (see MA94). They slightly differ from the genuine $Z R_{\mathrm{X}}$ and $Z R_{\mathrm{K}}$ results shown in upper panels. The two $R$ profiles for the DF algorithm are in excellent agreement $(\langle R\rangle \approx 5$ $\mathrm{mm} \mathrm{h} \mathrm{h}^{-1}$ below the bright band). Comparing with the $Z_{\mathrm{K}}^{\text {cor }}-R$ results at each frequency points out credible corrections of PIA effects.

\footnotetext{
${ }^{2}$ Superscript "cor" stands for the involved correction of $Z-R$ estimate.
} 

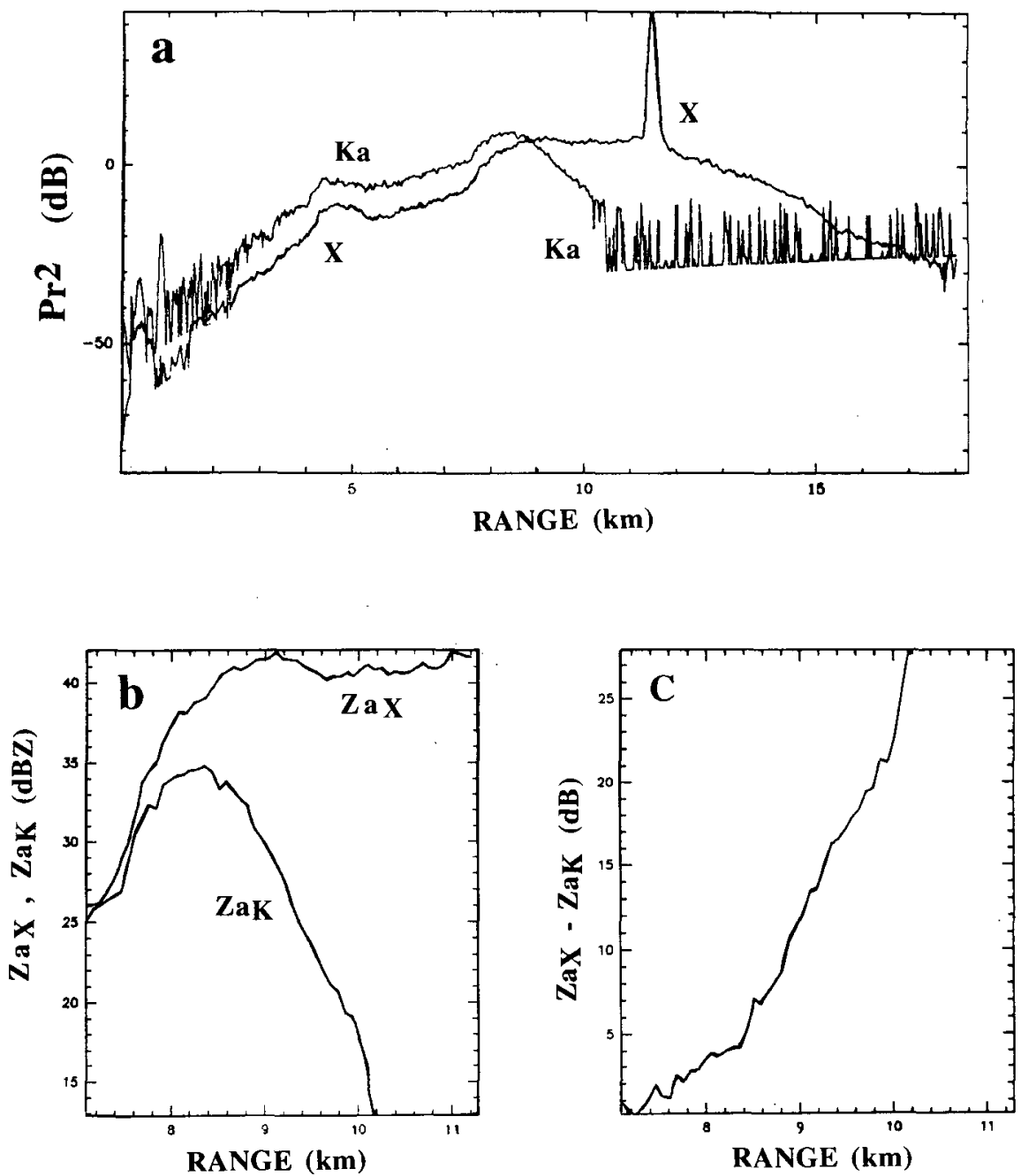

FIG. 7. Same as in Fig. 5 but for radar measurements within convective rain (observation 2233 , off-nadir beam incidence $6^{\circ}$ ).

The second example corresponding to observation 2233 in convective rain with high rain rate is displayed in Fig. 7. The range-normalized rain powers and the derived apparent reflectivities at both frequencies do not reveal the presence of bright band. At $\mathrm{K}_{\mathrm{a}}$ band, rain signal falls below noise at ranges beyond $10.2 \mathrm{~km}$, and the surface echo is not detected. The difference between both reflectivities increases up to $28 \mathrm{~dB}$ in the detection range. Results for the SF and DF algorithms are shown in Fig. 8. Compared with previous results for stratiform rain, the SF algorithm results (upper panels ) point out the increase in attenuation effects: PIA $\approx 3.8 \mathrm{~dB}$ and PIA $_{K}>40 \mathrm{~dB}$. At $X$ band, results for the various "attenuation" algorithms are scattered within a margin of about $50 \%$ near the surface. At $\mathrm{K}_{\mathrm{a}}$ band, $k Z$ algorithm results, close to $Z-R$ estimate, strongly underestimate the $R$ profile, while the nearly identical results for $k Z C_{\mathrm{K}}$ and $k Z S_{\mathrm{K}}$ algorithms diverge at low SNR near range $10 \mathrm{~km}$. The $k Z N_{\mathrm{K}}$ algorithm yields intermediate results. Corrections of scaling errors that could conciliate $R$ profiles from "attenuation" algorithms at $\mathrm{X}$ and $\mathrm{K}_{\mathrm{a}}$ band are approximately -1.2 $\mathrm{dB}$ for PIA $\mathrm{A}_{\mathrm{X}}$ in $k Z C_{\mathrm{X}}$ and $k Z S_{\mathrm{X}}$ and $-2.3 \mathrm{~dB}$ for the radar "calibration" in $k Z_{\mathrm{K}}$. Conversely, the two $R$ profile estimates produced by the DF algorithm are very similar over the common range interval where both rain signals are detected and are not affected by the detection limit at $\mathrm{K}_{\mathrm{a}}$ band. The $Z^{\text {cor }}-R$ results, at each frequency, are different by a factor up to 2 from those of the genuine $Z-R$ relations. In absence of such corrections, attenuation effects would appear unrealistically large, especially at $\mathrm{X}$ band.

\section{d. Statistical tests and $2 D$ rain structures from an ensemble of rain profiles}

We characterized the overall quality of the rain rates recovered from various algorithms by comparing the 

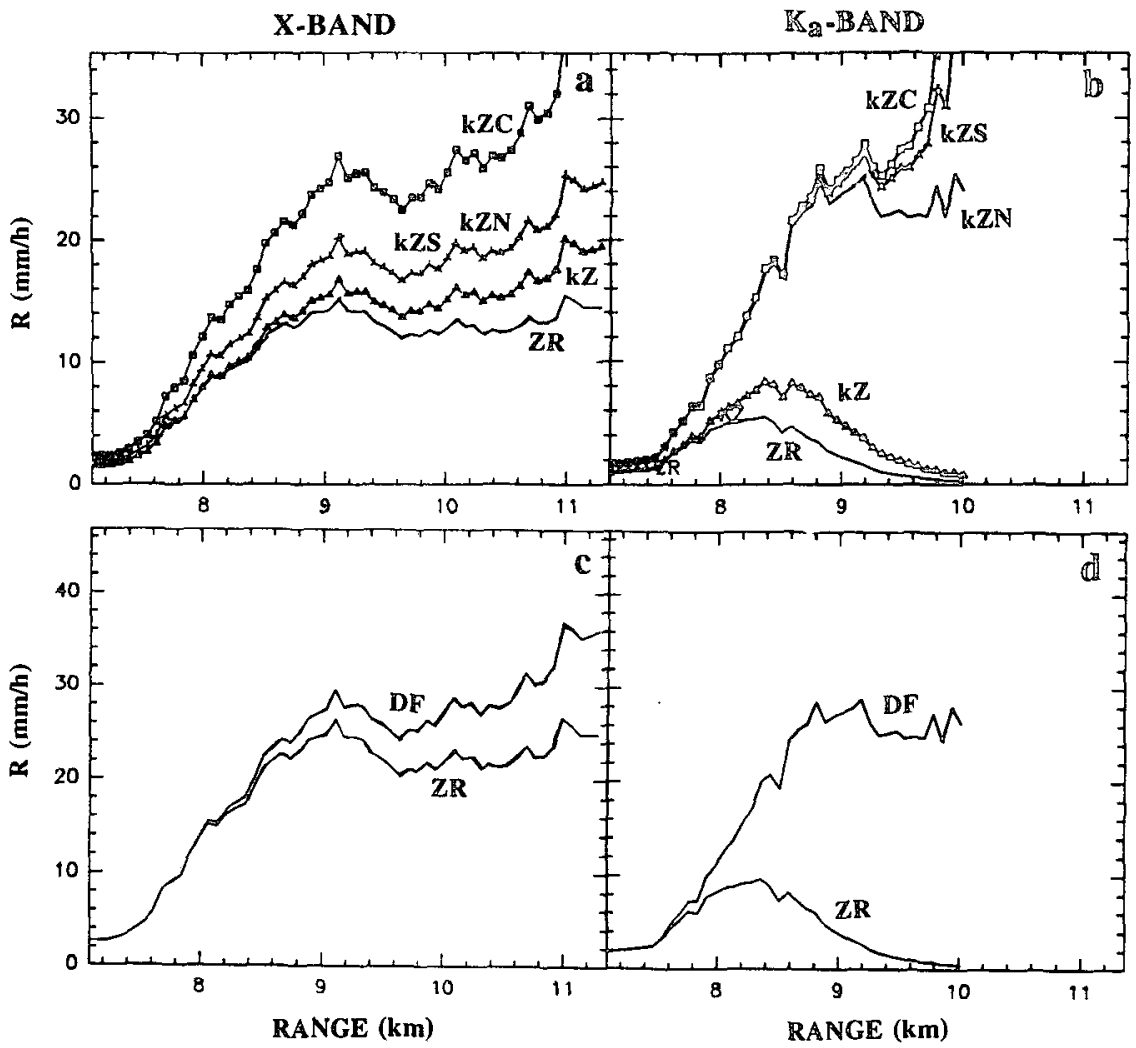

Fig. 8. Same as in Fig. 6 but for range-profiled rain rates retrieved within convective rain (observation 2233, off-nadir beam incidence $6.1^{\circ}$ ). See radar measurements in Fig. 7.

height-averaged rain rates computed from the $1601 R$ profiles of the dataset. For saving internal coherency of the results for stratiform and convective precipitation, the height-average $\langle R\rangle$ of the $R$ profiles was performed over the first $2.5 \mathrm{~km}$ above the surface, that is, within rain below the melting region. The results presented below are focused on $k Z S, D F$, and $Z-R$ algorithms.

The results for $k Z S$ and $Z-R$ algorithms are displayed in Fig. 9. At $X$ band, the two estimates are close to each other except for high rain rate where attenuation effects becomes significant. The $k Z S_{\mathrm{K}}$ algorithm performs large corrections of PIA effects, while $Z R_{\mathrm{K}}$ "saturates" above about $2 \mathrm{~mm} \mathrm{~h}^{-1}$. Results for $k Z S_{\mathrm{X}}$ and $k Z S_{\mathrm{K}}$ are fairly close to each other, which points out the capability of the $k Z S$ algorithm to correct for "attenuation" effects within a large dynamic range. The convergence of $k Z S$ algorithm results toward $Z R_{\mathrm{X}}$ estimates is well observed for low attenuations, that is, $\langle R\rangle$ below $10 \mathrm{~mm} \mathrm{~h}^{-1}$. At $\mathrm{K}_{\mathrm{a}}$ band, $k Z S_{\mathrm{K}}$ and $Z R_{\mathrm{K}}$ are close to each other for $\langle R\rangle$ below $2 \mathrm{~mm} \mathrm{~h}^{-1}$ only.

The same kind of results, but for the $k Z S$ and $k Z$ algorithms results, are displayed in Fig. 10. At X band, the two estimates are very close to each other. Differences between $k Z S_{\mathrm{X}}$ and $k Z_{\mathrm{X}}$ are generally smaller than between $k Z S_{\mathrm{X}}$ and $Z R_{\mathrm{X}}$ (see Fig. 9). Conversely, $k Z_{\mathrm{K}}$ produces unstable results that generally underestimate the rain rate except near observation 2270 where a local overestimation effect is observed. This confirms that $k Z$ algorithm corrects almost adequately low PIA effects $(<3 \mathrm{~dB})$ but fails to correct large PIA effects $(>3$ $\mathrm{dB}$ ); it again points out the benefit of using surface echo as a constraint to improve the results.

The two rain estimates derived from the DF algorithm, at $X$ band and at $K_{a}$ band, along with the mean rain rates using $Z^{\mathrm{cor}}-R$ relations at each frequency, are plotted in Fig. 11. We could verify that some isolated spikes were due to "bad" convergence of the DF algorithm. They correspond to cases where the required relative minimum of the functional used to adjust the $k\left(f_{2}\right)-k\left(f_{1}\right)$ relation in the nonlinear regression process (MA94) was very flat, or not even found. This happened for about $5 \%$ of the cases, especially for high rain rates. The general behaviors of the results from the $\mathrm{DF}$ algorithm and the $k Z S$ algorithm (shown in Fig. 9) are similar, despite some differences observed in the magnitudes of rain peaks. Also, the mean rain rates estimated either from the genuine $Z R_{\mathrm{X}}\left(Z R_{\mathrm{K}}\right)$ relation or from the "corrected" $Z_{\mathrm{X}}^{\text {cor }}-R\left(Z_{\mathrm{K}}^{\text {cor }}-R\right)$ relation may be different. This is the case at $\mathrm{X}$ band, near observations $3150-3200$ and 3350 , where $Z-R$, in contrast to $Z \underset{\mathrm{X}}{\text { cor }}-R$, produces questionable higher $\langle R\rangle$ estimates than DF algorithm. In Fig. 12 the two $\langle R\rangle$ es- 


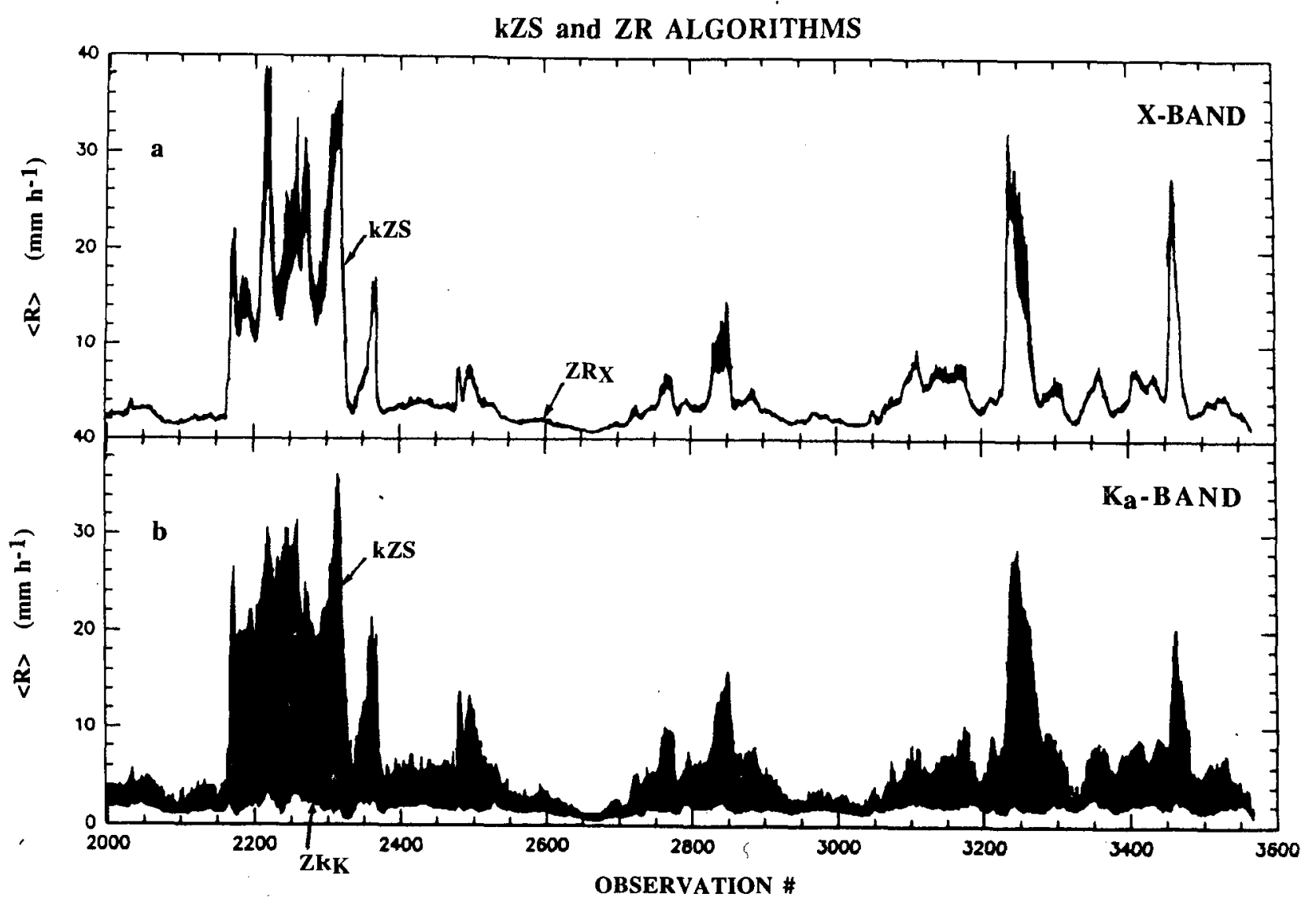

FIG. 9. Mean rain rates $\langle R\rangle$ (average over a $2.5-\mathrm{km}$ depth above the surface) computed from $1601 R$ profiles (observations $2000-3600$ on 1 November 1988) retrieved from $k Z S$ and $Z-R$ algorithms (a) at $X$ band and (b) at $K_{\mathrm{a}}$ band. For each panel, a positive (negative) difference between the $k Z S$ - and $Z-R$ algorithms results is characterized by a black (blank) area.

timates from the DF algorithm are plotted again, as is the fractional deviation with respect to the mean of the two estimates. Similarity of both estimates, which is a necessary condition to get reliable results, is generally observed. Sporadic deviations (e.g., near observation 3450) are above-mentioned cases of "bad" convergence.

Another convenient way to globally characterize the results is to analyze the correlation between the $\langle R\rangle$ results over the 1601 observations for different pairs of algorithms using scatterplots. Such plots are shown in Fig. 13. For each case, the correlation coefficient $\rho$ between $\left\langle R_{1}\right\rangle$ and $\left\langle R_{2}\right\rangle$ and the standard deviation (SD) relative to the mean of the two estimates are indicated in the legend. The line figuring out a hypothetical linear best fit to the results is also plotted.

Results at $K_{a}$ band (Fig. 13a) illustrate the extent of the dynamic range over which $k Z S_{\mathrm{K}}$ compensates for attenuation effects, while $Z R_{\mathrm{K}}$ saturates rapidly and underestimates the rainfall as soon as $\langle R\rangle$ exceeds about $1 \mathrm{~mm} \mathrm{~h}^{-1}$ in the present case. For low attenuation, convergence of both results toward the same values underlines the stability of $k Z S_{\mathrm{K}}$ and its complementarity with $Z-R$. The same effect is observed for $k Z S_{\mathrm{X}}$ (Fig. $13 \mathrm{~b}$ ), but the threshold over which $Z R_{\mathrm{X}}$ is negatively biased is increased to about $12 \mathrm{~mm} \mathrm{~h}^{-1}$. The $k Z S_{\mathrm{X}}$ and $k Z S_{\mathrm{K}}$ results (Fig. 13c) are in fair agreement except for low rain rates $\left(<10 \mathrm{~mm} \mathrm{~h}^{-1}\right)$, where $k Z S_{\mathrm{X}}$ tends to produce lower estimates. An agreement between $k Z S_{\mathrm{K}}$ and DF algorithm results (Fig. 13d) is acceptable, although significant scatter is observed. Indeed, agreement is not expected to be perfect since the DF algorithm is potentially able to perform better attenuation corrections than $k Z S$ algorithm except for some "bad convergence" cases alluded to earlier.

Finally, Fig. 14 shows several reconstructions of a 2D cross section of the rain rate from various algorithms within a part of the precipitating system. The selected part of the system corresponds to 201 observations (observations 2800 to 3000 ), or about $16 \mathrm{~km}$ in length. The rain-rate structures were drawn from gridded $R$-profiles results with a vertical resolution of $300 \mathrm{~m}$, and an "equivalent" along-track resolution of $640 \mathrm{~m}$ using running mean over eight contiguous observations. 
$k Z S$ and $k Z$ ALGORITHMS

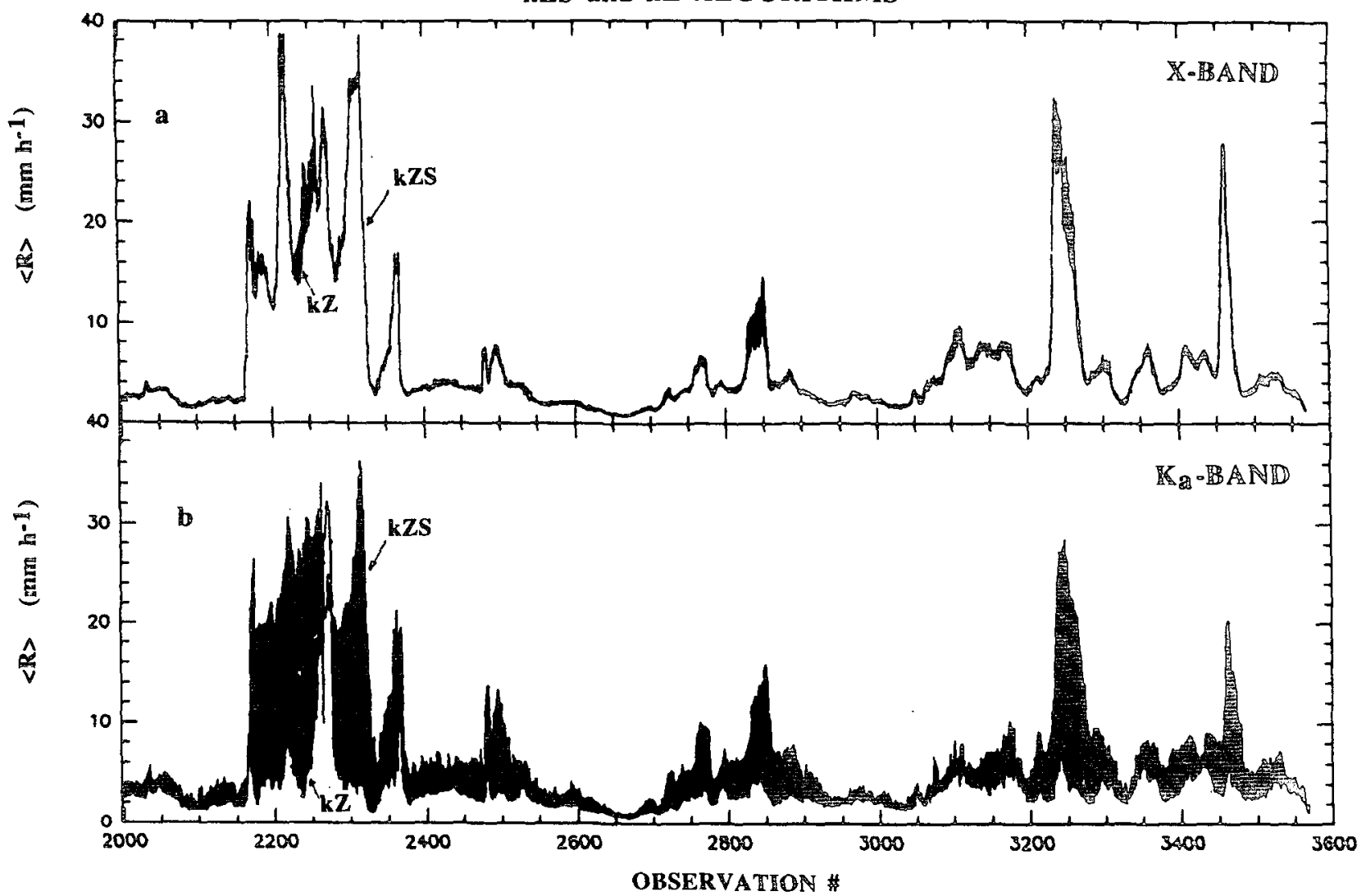

FiG. 10. Same as in Fig. 9 but for the rain estimates provided by the $k Z S$ and $k Z$ algorithms, (a) at $\mathrm{X}$ band and (b) at $\mathrm{K}_{\mathrm{a}}$ band.

Results from $Z R_{X}$ (Fig. 13a) point out well-defined rain structures with a rain-cell core $\left(R_{\max } \approx 9 \mathrm{~mm} \mathrm{~h}^{-1}\right)$ centered at abscissa $x=4 \mathrm{~km}$ and a brightband peaking aloft near $3-\mathrm{km}$ altitude, in the presence of moderate PIA effects. Almost all the observed structure are scratched in $Z R_{\mathrm{K}}$ estimate (Fig. 13b), owing to uncorrected PIA effects severely increasing deep in the rain. Attenuation corrections produced by $k Z S_{X}$ (Fig. $13 \mathrm{c}$ ) and $k Z S_{\mathrm{K}}$ (Fig. 13d) lead to fair recoveries of rain-rate patterns $\left(R_{\max } \approx 15-18 \mathrm{~mm} \mathrm{~h}^{-1}\right)$, especially at X band, with brightband peak much weaker at $K_{\mathrm{a}}$ band than at $\mathrm{X}$ band. The DF algorithm results yield the most detailed structures in rain $\left(R_{\max } \approx 18 \mathrm{~mm} \mathrm{~h}^{-1}\right)$ and in the melting region. Below $2-\mathrm{km}$ altitude, and for abscissas between 2 and $4 \mathrm{~km}$, the recovered $2 \mathrm{D}$ rain structure combines some local features obtained in $k Z S_{\mathrm{X}}$ and $k Z S_{\mathrm{K}}$ results, respectively. Let us recall that the $k Z S$ algorithm is constrained by surface echo measurements, while the DF algorithm does not make an explicit use of such additional measurements. Use of $\mathrm{K}_{\mathrm{u}}$ band (near $14 \mathrm{GHz}$ ), or $\mathrm{K}$ band (near $24 \mathrm{GHz}$ ), is more commonly envisioned for spaceborne radars, such as TRMM radar at $K_{\mathrm{u}}$ band (Simpson et al. 1988). Results of $Z-R$ algorithm would be intermediate from those obtained at X and $\mathrm{K}_{\mathrm{a}}$ bands, while the SF and DF algorithms, correcting for the involved PIAs, would likely yield similar kinds of results. For the DF algorithm, however, some features in the results may more specifically depend on the selected frequency pair, as suggested in MA94.

\section{Simulation of morraminiform beam-hilling efiecte}

NUBF effects induced by rain-rate gradients within the radar beamwidth may be a significant source of bias in rain retrieval from spaceborne radar measurements. Very few studies have used real data precipitation fields to simulate such effects. Examples of works involving simulated spatial rain structures, such as models of rain cell defined on theoretical basis or derived from observed rainfall statistics, are more numerous (e.g., Goldhirsh and Walsh 1982; Testud et al. 1992; Amayenc et al. 1993; among others). These approaches allow an extended choice of simulation conditions but they are limited because realistic rain-rate distributions at various space scales are difficult to model. Two main ways may be followed to study NUBF effects taking into account actually observed rain structures.

A first way is to use precipitation fields derived from radar observations at nonattenuating frequencies. Na- 


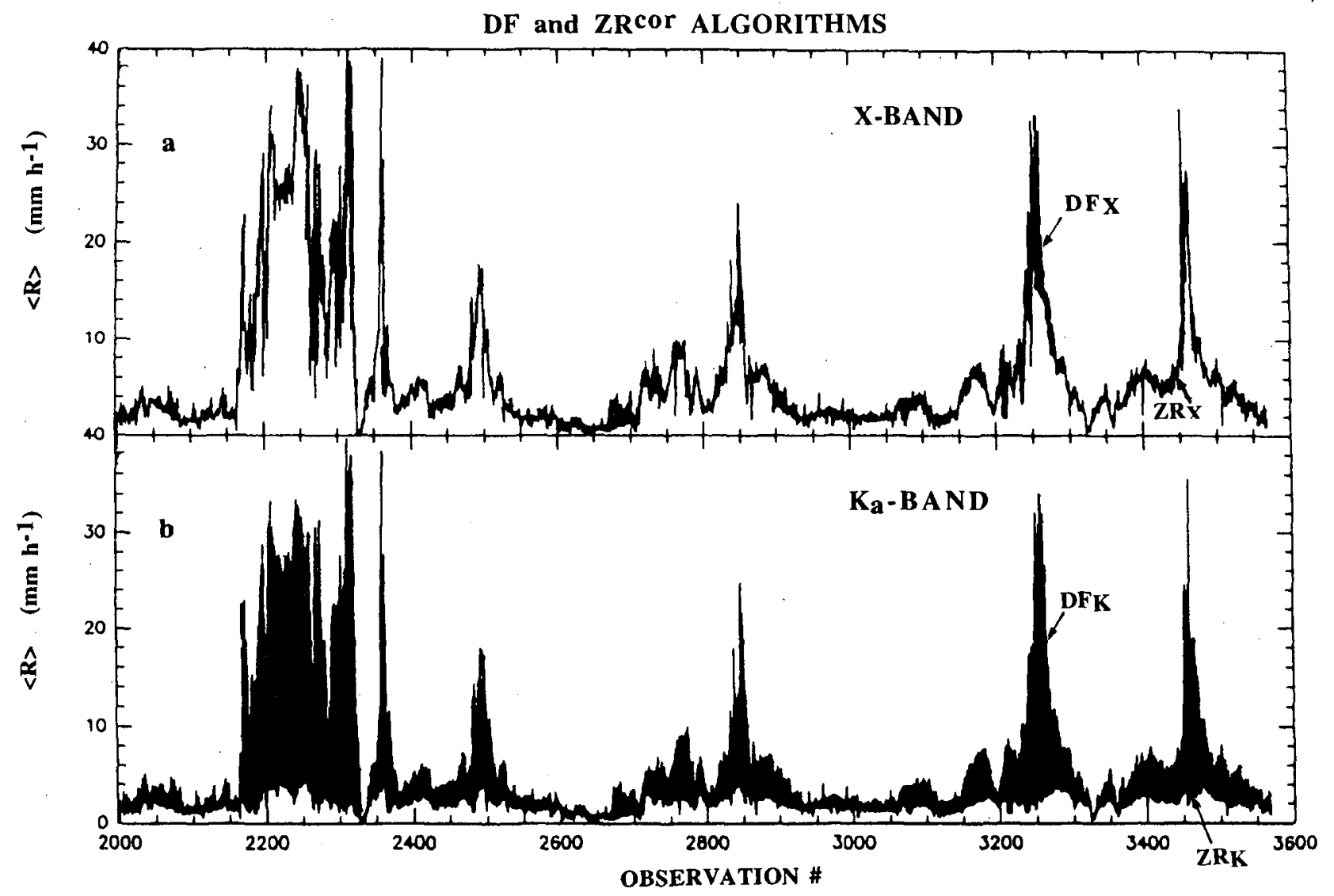

FiG. 11. Same as in Fig. 9 but for the two rain estimates provided by the DF algorithm, (a) at $X$ band and (b) at $K_{a}$ band, and for the rain estimate provided by the corrected $Z^{\mathrm{cor}}-R$ algorithm at each frequency.

kamura (1991) used a 1D time series of X-band radar measurements in Japan at a slant range of $2 \mathrm{~km}(1-\mathrm{km}$ altitude). He assumed no height variation of the rain rate to produce a $2 \mathrm{D}$ rain field within an equivalent vertical plane. Then he used this rain field to simulate effects of NUBF in spaceborne radar measurements for different cross-range resolutions of a nadir-pointing radar beam and for several rain retrieval algorithms.

A second way is to use airborne radar observations simulating spaceborne measurements at attenuating frequencies. This is the method used in the present paper. Let us recall, however, that most conditions of the simulation are inherently attached to the measurements characteristics. Here results refer to the frequencies 10 and $35 \mathrm{GHz}$ and to near-nadir beam incidence over ocean. In addition, since no acrosstrack information is available in the data, study of NUBF effects may be performed in $2 \mathrm{D}$ frame only.

\section{a. Simulation conditions}

\section{1) Method and data For BEAM aVERAGing}

As explained in section $3 b$, the radar provides a nearly vertical cross section of the overflown rainy sys- tem. The sampling step is $\delta x \approx 80 \mathrm{~m}$ in the alongtrack direction, denoted $x$. Every observation is obtained with a cross-range resolution $L_{0} \approx 1 \mathrm{~km}$ near the surface, equivalent to about 12 observations, and $L_{0} \approx 0.5$ $\mathrm{km}$ at $5.6-\mathrm{km}$ height (half the mean flight altitude) equivalent to about 6 observations. Thus, for each range gate, we are facing the following situation.

(i) Every genuine radar measurement may include some beam-averaging effect at " $L_{0}$ scale" involving weighted contribution of horizontal rain structures within an along-track distance interval $\pm L_{0} / 2$, equivalent to \pm 6 observations next to the surface, on each side of the measurement in question.

(ii) There is a large overlap from every observation to the next one.

(iii) Nearly independent observations corresponding to contiguous footprints, according to a sampling interval $L_{0} \approx 1 \mathrm{~km}$, are obtained every 12 observations next to the surface.

Our purpose is to use this configuration to infer NUBF effects for cross-range resolutions $L>L_{0}$. Though NUBF effects are likely small at $L_{0}$ scale, the small sampling step $\left(\delta x \ll L_{0}\right.$ ) prevents us from con- 

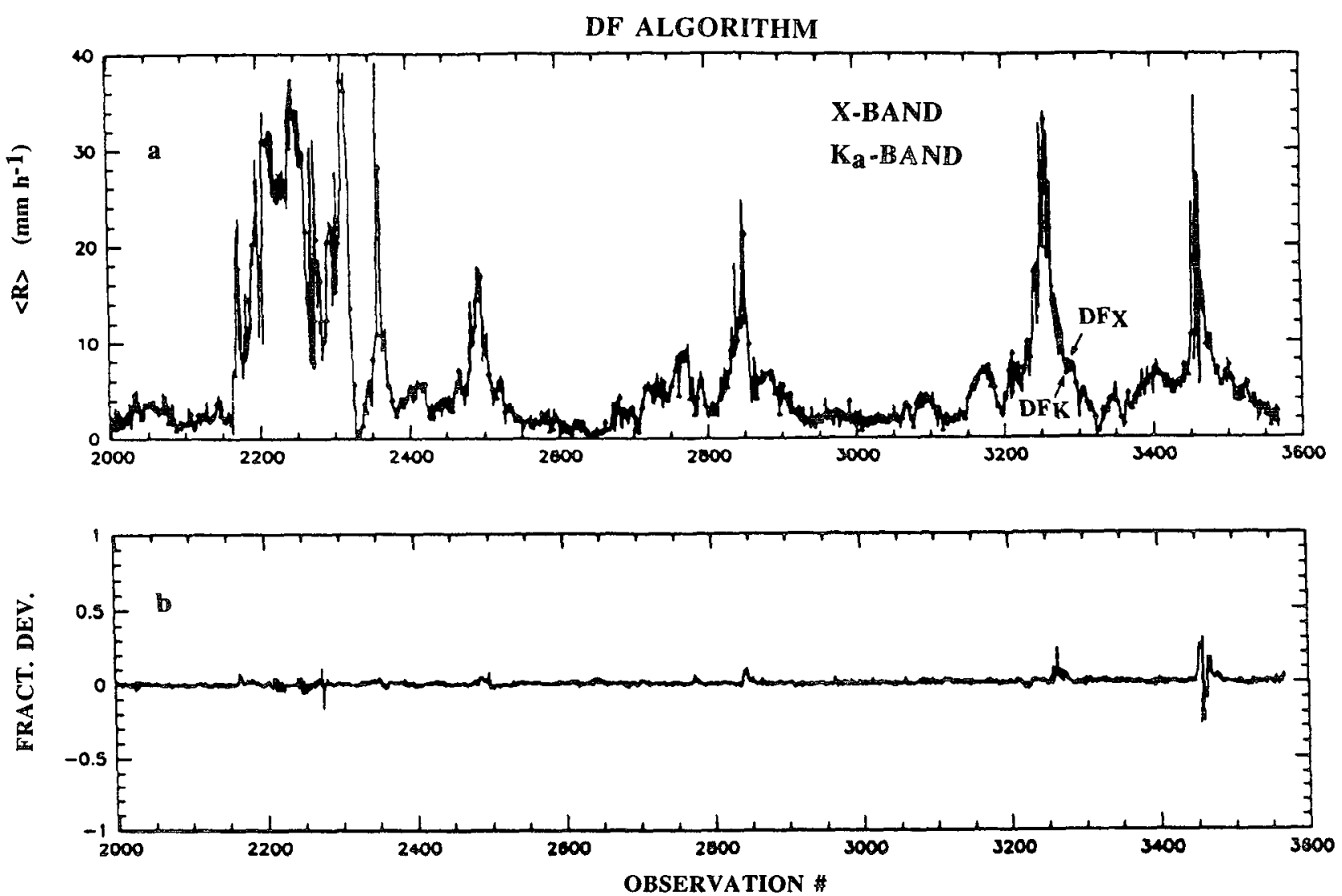

FIG. 12. Same as in Fig. 11 but (a) for the two rain estimates provided by the DF algorithm and (b) for their fractional deviation with respect to the mean.

sidering that radar measurements for successive observations are independent. Thus, we cannot compute a simple average, as if data were obtained with resolution $\delta x$, to get beam-averaged quantities for $L>L_{0}$. In fact, a correct simulation of NUBF effects at " $L$ scale" should first involve a deconvolution of beam-averaging at $L_{0}$ scale to get measurements at " $\delta x$ scale," then a new beam-averaging at $L$ scale, for each altitude. Such a deconvolution, however, which would require an accurate knowledge of the radar beam gain function, is hard to conceive in the presence of path attenuation. Besides, it is generally unstable and vulnerable to noise. Thus, we looked for a more simple approach while assuming the following "idealized" conditions, which fit rather well to the observation.

(i) Measurements are obtained with a constant aircraft flight altitude and a nadir-pointing radar beam.

(ii) Resolution $L_{0}$ is taken constant with range and is equal to the value $(1 \mathrm{~km})$ near the surface.

(iii) The selected values $L=2,3$, and $4 \mathrm{~km}$, also assumed constant with range, are typical of those actually envisioned for spaceborne radars; $L=4 \mathrm{~km}$ is close to the TRMM radar resolution at nadir. (iv) The gain function of the simulated radar beam is Gaussian shaped.

(v) NUBF effects are mostly produced by alongtrack rain structures within the $\pm L / 2$ distance interval and neglected in the acrosstrack direction.

The beam-averaged measurements at $L$ scale were performed by a combining beam-averaged measurements at $L_{0}$ scale according to

$$
X_{m L_{0}}\left(N_{0}\right)=\sum_{n=N_{0}-6 m}^{n=N_{0}+6 m} X_{L_{0}}(n)\left[\frac{W_{\mathrm{G}}^{2}(n)}{P_{w}}\right] g\left(n-N_{0}\right),
$$

where $X$ is the averaged quantity at a given range (see below), $X_{L}$ denotes beam-averaged value of $X$ at $L$ scale, $m=L / L_{0}$ refers to beam-averaging at " $m L_{0}$ scale," that is, $L$ scale, $N_{0}$ is the reference observation number of the averaged quantity, $g\left(n-N_{0}\right)=1$ for $\left(n-N_{0}\right)=6 k$ ( $k$ is an integer $)$ and $=0$ otherwise, $W_{\mathrm{G}}$ is the weighting factor of a Gaussian-shaped beam gain function given by

$$
W_{\mathrm{G}}(n)=\exp \left\{-4(\ln 2)\left[\frac{\left(n-N_{0}\right) \delta x}{L}\right]^{2}\right\}
$$



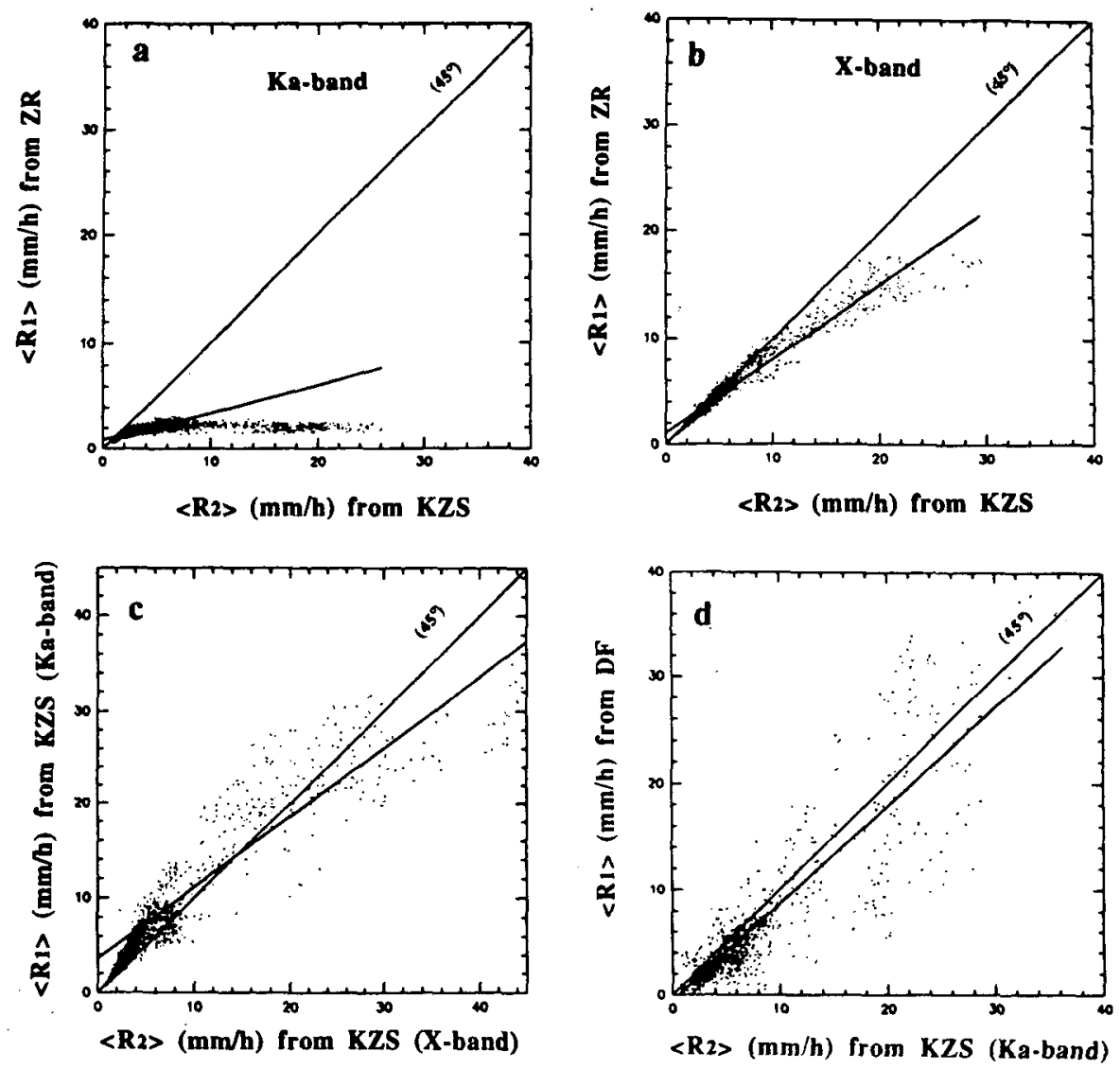

Fig. 13. Scatterplots of mean rain-rate estimates (average over a $2.5 \mathrm{~km}$ depth above the surface) $\left\langle R_{1}\right\rangle$ versus $\left\langle R_{2}\right\rangle$ computed from $1601 R$ profile pairs (observations $2000-3600$ on 1 November 1988 ) recovered from various algorithms. The involved algorithms for $\left\langle R_{1}\right\rangle$ and $\left\langle R_{2}\right\rangle$ are (a) $Z-R$ versus $k Z S$ at $\mathrm{K}_{\mathrm{a}}$ band $(\rho=0.825, \mathrm{SD}=70 \%)$; (b) $Z-R$ versus $k Z S$ at $\mathrm{X}$ band $(\rho=0.976, \mathrm{SD}=14 \%)$; (c) $k Z S$ at $\mathrm{K}_{\mathrm{a}}$ band versus $k Z S$ at $\mathrm{X}$ band $(\rho=0.902, \mathrm{SD}=29 \%)$; (d) DF versus $k Z S$ at $\mathrm{K}_{\mathrm{a}}$ band $(\rho=0.902$, $\mathrm{SD}=41 \%)$. The bisectrix $\left(45^{\circ}\right.$ line $)$ and the linear regression line best fitting the data are also indicated for each case.

$\hat{s} x=L_{0} / 12(\approx 80 \mathrm{~m})$, and $P_{W}$ is a normalizing factor of the gain function, such as $\Sigma W_{\mathrm{G}}^{2} / P_{w}=1$, given by

$$
P_{W}=\sum_{n=N_{0}-6 m}^{n=N_{0}+6 m} W_{\mathrm{G}}^{2}(n) g\left(n-N_{0}\right) \text {. }
$$

The scheme for beam-averaging using (4) is illustrated in Fig. 15. Beam-averaging at $L$ scale is performed by using beam-averaged measurements at $L_{0}$ scale with a step $L_{0} / 2$. This value was chosen as a tradeoff between the following two extremes cases: (i) a step $L_{0}$, for contiguous measurements at $L_{0}$ scale, which could lead to lose some rain structures contributing to the beam filling; (ii) a step $\delta x$ that would risk overaveraging rain structures, which would contribute to the beam filling. The method actually performs a $2 \mathrm{D}$ simulation of beam averaging within along-track-vertical space frame. In genuine data, the dimension of the "circular" 3-dB footprint is $L_{0}=1 \mathrm{~km}$. In the simulations, the "equivalent" dimensions of the 3-dB footprint are
$L_{0}=1 \mathrm{~km}$ across-track and $L=2,3$, or $4 \mathrm{~km}$ alongtrack, respectively.

We used the same dataset of 1601 observations, as in section $4 \mathrm{~d}$. For every selected observation $N_{0}$, we performed beam averaging at $L$ scale of the range-gated received powers, including rain and surface returns at each frequency. In regions where signal was not detected, we left the noise to be summed up in the averaging process. By calculating the involved quantities at the "genuine'" $L_{0}$ scale, that is, using (4) to recalculate $X_{L_{0}}$ and comparing with the original results, we verified that the procedure did not induce bias due to some overaveraging effect. The $\sigma^{0}$ model outside rain was found to change by less than $0.2 \mathrm{~dB}$ at both frequencies for $L$ $=2,3$, or $4 \mathrm{~km}$, likely as a result of low variability in $\sigma^{0}$ at $L_{0}$ scale.

\section{2) TESTS USED FOR ANAL YZING NUBF EFFECTS}

The sound nature of NUBF effects and the way they may be characterized have to be discussed. In essence, 

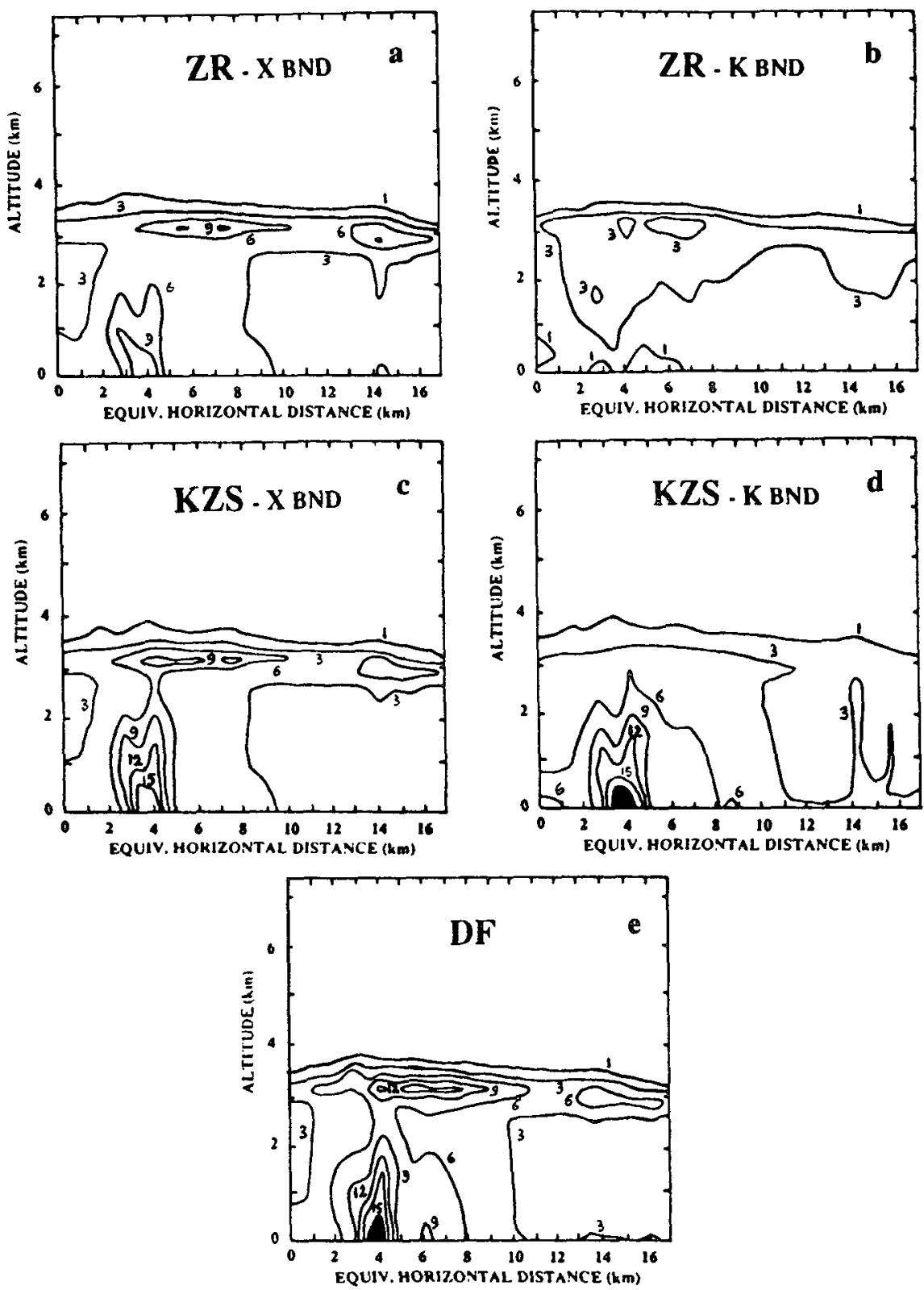

FIG. 14. Vertical cross sections (altitude versus the equivalent horizontal distance) of rain-rate structures reconstructed from the results of various range-profiling algorithms: (a) $Z-R$ algorithm at $\mathrm{X}$ band, (b) $Z-R$ algorithm at $\mathrm{K}_{\mathrm{a}}$ band, (c) $k Z S$ algorithm at $\mathrm{X}$ band, (d) $k Z S$ algorithm at $\mathrm{K}_{\mathrm{a}}$ band, and (e) DF algorithm. For each case, the results involve 201 contiguous recovered $R$ profiles (observations $2800-3000$ on 1 November 1988) at nearly vertical incidence.

given an algorithm and a cross-range resolution $L$, such effects refer to possible difference between the retrieved rain rate $R_{L}$ and the "true" mean rain rate $R_{L}^{*}$ averaged over $L$, which is the quantity actually expected to be retrieved.

When using theoretical rain models, it is possible to compute directly $R_{L}^{*}$. Analytical results and numerical simulations with $k Z S$ and $k Z$ algorithms (Testud et al.
1992; Amayenc et al. 1993) showed that $R_{L}$ generally underestimates the "true" mean rain rate $R_{L}^{*}$, owing to NUBF effects increasing with $L$. From simulations with real data, Nakamura (1991) found that either underestimation or overestimation effects, increasing with $L$, may occur depending on the involved algorithm.

When using real data from airborne radar, $R_{L}^{*}$ is not known since the available rain-rate estimate $R_{L}$ is de- 


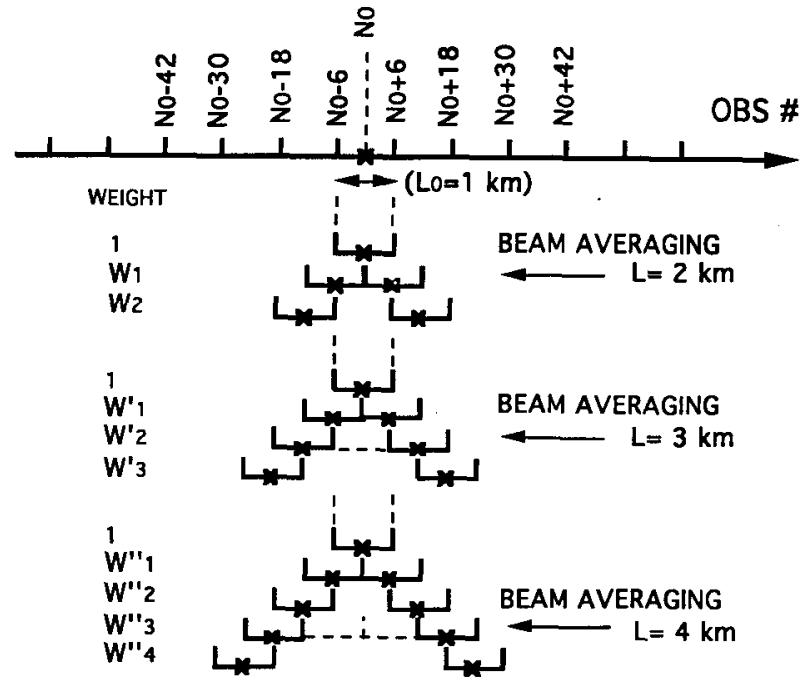

FIG. 15. Schemes used to simulate beam averaging for the selected 3-dB cross-range resolutions $L=2,3$, or $4 \mathrm{~km}$ from the genuine measurements with $3-\mathrm{dB}$ cross-range resolution $L_{0}=1 \mathrm{~km}$. All schemes refer to observation $N_{0}$. Crosses $(\times)$ figure out the observations involved in the procedure. The normalized weighting factors, indicated qualitatively on the left-hand side, identify with the term $W_{G}^{2} / P_{w}$ in Eq. (4); they correspond to a Gaussian-shaped gain function for a cross-range resolution $L$ and apply to the involved observations on the same line.

rived from the results of the algorithms themselves. Given an algorithm, it is, however, possible to compare the rain rates $R_{L}$ for different values of $L$. But if $R_{L_{0}}$ is taken as reference, there is no theoretical reason to expect that similar characteristics be obtained by comparing $R_{L}$ (for $L>L_{0}$ ) and $R_{L_{0}}$ or $R_{L}$ and $R_{L}^{*}$. A way to improve the conditions of comparison is to look for an estimate of $R_{L}^{*}$ elaborated from $R_{L_{0}}$, which is the only available experimental quantity. Accordingly, the true mean rain rate $R_{L}^{*}$ was approximately computed from a weighting average of contiguous $R_{L_{0}}$ values over the cross-beam resolution $L$ in question for every reference observation $N_{0}$, according to the general scheme

$$
R_{L}^{*}\left(N_{0}\right)=\frac{1}{m} \sum_{n=N_{0}-6(m-1)}^{n=N_{0}+6(m-1)} R_{L_{0}}(n) h\left(n-N_{1}\right)
$$

where $N_{1}=N_{0}-6(m-1), L=m L_{0}$ with $m=1,2$, 3,4 , and $h\left(n-N_{1}\right)=1$ for $\left(n-N_{1}\right)=12 k(k$ is integer of at least 0 ) and $=0$ otherwise.

Equation (7) verifies $R_{L}^{*}\left(N_{0}\right)=R_{L_{0}}\left(N_{0}\right)$ for $L=L_{0}$. As an example for $L=3 L_{0}$, the computation scheme is

$$
\begin{aligned}
R_{L}^{*}\left(N_{0}\right)=\frac{1}{3}\left[R_{L_{0}}\left(N_{0}-12\right)+\right. & R_{L_{0}}\left(N_{0}\right) \\
& \left.+R_{L_{0}}\left(N_{0}+12\right)\right] .
\end{aligned}
$$

The $R_{L}^{*}$ estimate depends on the considered algorithm. This is, however, not very penalizing because $R_{L_{0}}$, even possibly different for the various algo- rithms, is weakly affected by NUBF. Also, it can be verified that for every algorithm the mean value [ $\left.R_{L}^{*}\right]_{N}$ of $R_{L}^{*}$ over a set of $N$ contiguous observations is independent of $L$ and nearly equal to the mean value of $R_{L_{0}}$; that is,

$$
\left[R_{L}^{*}\right]_{N} \approx \frac{1}{N} \sum_{n=1}^{n=N} R_{L_{0}}(n),
$$

provided that $N \gg 6(m-1)$. A small difference may appear, resulting from edge effects.

In the following, we present results of the comparison between $R_{L}$ (the $3-\mathrm{dB}$ beam-averaged rain rate at $L$ scale) and either $R_{L_{0}}$ (the 3-dB beam-averaged rain rate at $L_{0}$ scale) or $R_{L}^{*}$ (the estimated true mean rain rate at $L$ scale) for every algorithm. The former comparison points out bulk effects of beam-averaging, while the latter one characterizes intrinsic effects of NUBF.

\section{b. Analysis of results}

We characterized beam-averaging effects in the height-averaged rain rates $\left\langle R_{L}\right\rangle$ computed from the $R_{L}$ profiles recovered from the algorithms over the 1601 observations of the dataset. The height average was still performed over the first $2.5 \mathrm{~km}$ above the surface. $\mathrm{Re}$ sults are focused on $k Z S, D F$, and $Z-R$ algorithms.

An example of simulated results, for $L=3 \mathrm{~km}$, are displayed in Fig. 16 for $Z_{\mathrm{X}}-R$ and $k Z S_{\mathrm{X}}$ and for $Z_{\mathrm{K}}-R$ and $k Z S_{K}$. They can be compared with those of Fig. 9 at $L_{0}$ scale $(1 \mathrm{~km})$. Similar results, for $L=3 \mathrm{~km}$, but referring to the two rain rate estimates (at $\mathrm{X}$ and $\mathrm{K}_{\mathrm{a}}$ band) of the DF algorithm, are plotted in Fig. 17, along with results obtained from $Z^{\text {cor }}-R$ relation at each frequency. They can be compared with those of Fig. 11 at $L_{0}$ scale. Beamaveraging effects are characterized by the following features in $\left\langle R_{L}\right\rangle$, compared with $\left\langle R_{L_{0}}\right\rangle$ :

(i) the "small scale" space structures of the rain rate are smoothed out;

(ii) $R_{L}$ generally decreases when $L$ increases for heavy rain and remains nearly unchanged for low rain; and

(iii) the most important changes are observed for regions that include large rain-rate gradients over a series of contiguous observations where local increase of $\left\langle R_{L}\right\rangle$ with $L$ may occasionally take place.

These features are valid for $k Z S$ and DF algorithms as well as for the $Z-R$ and $Z^{\text {cor }}-R$ algorithms results; they strengthen with $L$ values increasing from 2 to 4 $\mathrm{km}$ (not shown). It is not surprising that increasing the averaging scale $L$ produces a decrease of $\left\langle R_{L}\right\rangle$. This effect is more pronounced for high rain rates that correspond to convective rain with nonhomogeneous structures at small scale than for low rain rates that correspond to more stratiform rain. Intrinsic NUBF effects, however, are present only if $R_{L}$ differs from $\left\langle R_{L}^{*}\right\rangle$ at $L$ scale. 


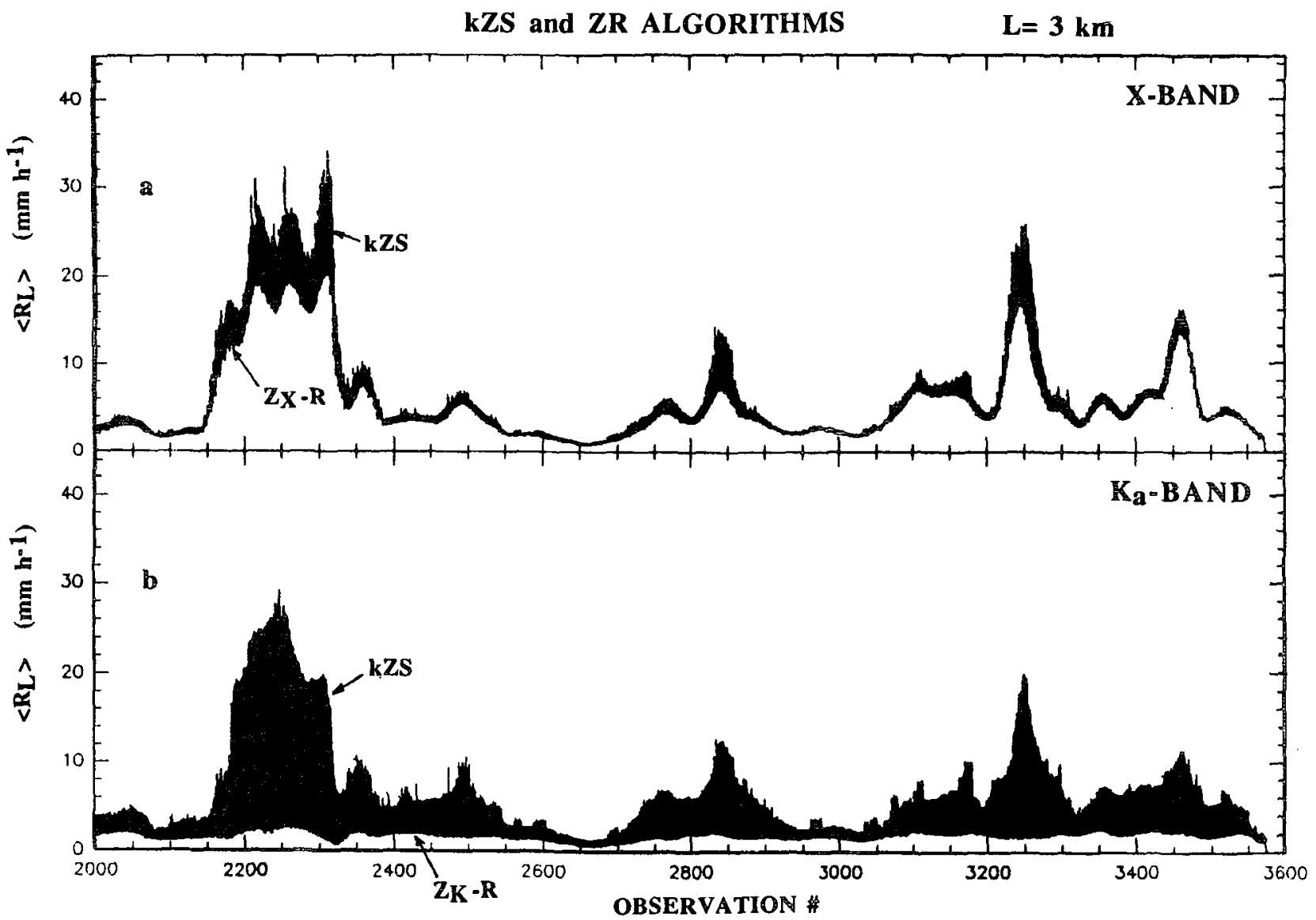

Fig. 16. Mean rain rates $\left\langle R_{L}\right\rangle$ (average over a $2.5-\mathrm{km}$ depth above the surface) computed from 1601 profiles (observations $2000-3600$ on 1 November 1988) retrieved from $k Z S$ and $Z-R$ algorithms with a simulated cross-range resolution $L=3 \mathrm{~km}$ : (a) at $X$ band and (b) at $\mathrm{K}_{\mathrm{a}}$ band. For each panel, a positive (negative) difference between $k Z S$ and $Z R$ algorithms results is characterized by a black (blank) area. See Fig. 9 for comparison with results at the original resolution $L_{0}=1 \mathrm{~km}$.

The correlation coefficient $\rho_{1}$ and the relative rms deviation $\sigma_{\text {Irms }}$ between $\left\langle R_{L}\right\rangle$ and $\left\langle R_{L_{0}}\right\rangle$ on one hand and $\rho_{2}$ and $\sigma_{2 \mathrm{rms}}$ between $\left\langle R_{L}\right\rangle$ and $\left\langle R_{L}^{*}\right\rangle$ on the other hand, as function of $L$, are listed in Table 4 for the $k Z S_{\mathrm{X}}$, $k Z S_{\mathrm{K}}$, and DF algorithms. The rms deviations, for the $N=1601$ observations were computed as

$$
\begin{aligned}
\sigma_{1 \mathrm{rms}} & =100\left\{\frac{1}{N} \sum_{n=1}^{n=N}\left(\frac{\left\langle R_{L}(n)\right\rangle-\left\langle R_{L_{0}}(n)\right\rangle}{\left\langle R_{L_{0}}(n)\right\rangle}\right)^{2}\right\}^{1 / 2} \\
\sigma_{2 \mathrm{rms}} & =100\left\{\frac{1}{N} \sum_{n=1}^{n=N}\left(\frac{\left\langle R_{L}(n)\right\rangle-\left\langle R_{L}^{*}(n)\right\rangle}{\left\langle R_{L}^{*}(n)\right\rangle}\right)^{2}\right\}^{1 / 2} .
\end{aligned}
$$

Results of Table 4 show that for every algorithm the rms deviation due to specific effects of NUBF ( $\left.\sigma_{2 \mathrm{rms}}\right)$ is greater than the rms deviation due to effects of beamaveraging $\left(\sigma_{1 \mathrm{rms}}\right)$, both increasing with $L$. A significant part of the observed deviations corresponds to bias in $R_{L}$ compared with either $R_{L}^{*}$ or $R_{L_{0}}$.
Finally, the impact of NUBF effects may be synthesized by comparing the averaged values of $\left\langle R_{L}\right\rangle$ and $\left\langle\dot{R}_{L}^{*}\right\rangle$ over the entire dataset. This kind of computation is important since a major goal for future spaceborne missions carrying rain radar is to provide rain-rate estimates at large scale. Let us recall that the 1601 observations are equivalent to an alongtrack length of about $128 \mathrm{~km}$. The mean rain rates over the dataset, denoted by the square brackets, were computed as

$$
\begin{aligned}
{\left[\left\langle R_{L}\right\rangle\right] } & =\frac{1}{N} \sum_{n=1}^{n=N}\left\langle R_{L}(n)\right\rangle \\
{\left[\left\langle R_{L}^{*}\right\rangle\right] } & =\frac{1}{N} \sum_{n=1}^{n=N}\left\langle R_{L}^{*}(n)\right\rangle .
\end{aligned}
$$

The variations of $\left[\left\langle R_{L}\right\rangle\right]$ versus $L$ for the various algorithms are plotted in Fig. 18a. The $\left[\left\langle R_{L_{0}}\right\rangle\right]$ estimates are close to each other for the "attenuation" algorithms. For $L$ ranging from 2 to $4 \mathrm{~km}$, all results stand within a $12 \%$ margin. The $Z R_{\mathrm{X}}$ algorithm results, moderately affected by uncorrected attenuation effects, 


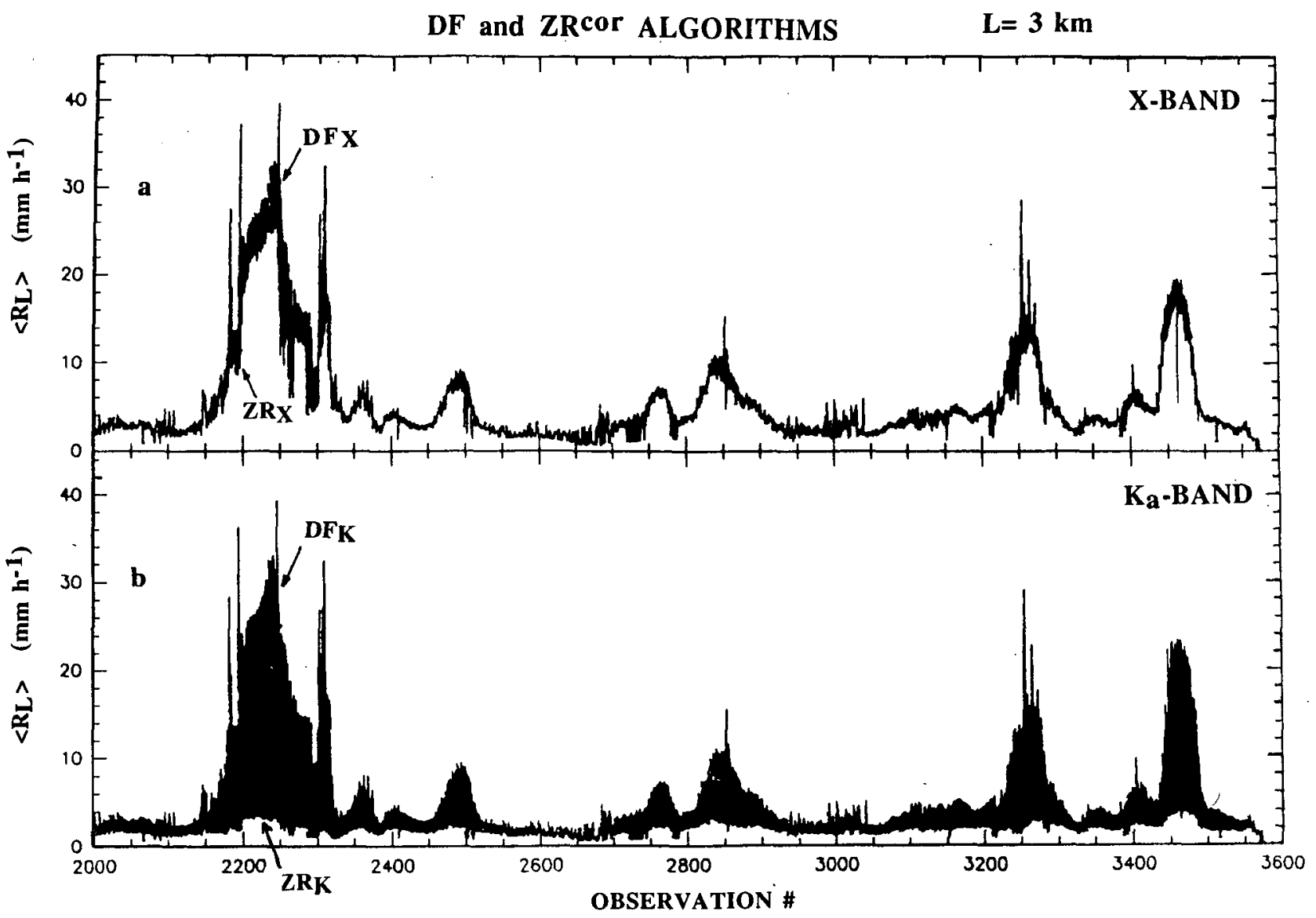

FIG. 17. Same as in Fig. 16 but for the DF algorithm and the "corrected" $Z$ cor $-R$ algorithm with a simulated cross-range resolution $L=3 \mathrm{~km}$ (a) at $\mathrm{X}$ band and (b) at $\mathrm{K}_{\mathrm{a}}$ band. See Fig. 11 for comparison with results at the original resolution $L_{0}=1 \mathrm{~km}$.

are $20 \%-30 \%$ lower. The $Z R_{\mathrm{K}}$ algorithm results, uncorrected for large PIA effects, are about $75 \%$ lower.

The variations of $\left[\left\langle R_{L}\right\rangle\right]\left[\left\langle R_{L}^{*}\right\rangle\right]^{-1}$ versus $L$, characterizing mean bias due to NUBF for the same algorithms, are plotted in Fig. $18 \mathrm{~b}$. Term $Z R_{\mathrm{X}}$, although it underestimates the rain rate, is almost unbiased (less than $2 \%$ ) by NUBF effects. The same is true for $Z R_{\mathrm{K}}$ results (not shown). For $k Z S_{\mathrm{X}}$, low positive bias due to NUBF slightly increases with $L$ from $+2 \%$ for $L=2$ $\mathrm{km}$ to $+4 \%$ for $L=4 \mathrm{~km}$. For $k Z S_{\mathrm{K}}$, negative bias due to NUBF increases with $L$ from $-8 \%$ for $L=2 \mathrm{~km}$ to $-12 \%$ for $L=4 \mathrm{~km}$. The DF algorithm results also indicate that negative bias due to NUBF increases with $L$ from $-12 \%$ for $L=2 \mathrm{~km}$ to $-15 \%$ for $L=4 \mathrm{~km}$. For every "attenuation" algorithm, the systematic difference (or bias) at $L$ scale between $\left[\left\langle R_{L}\right\rangle\right]$ and $\left[\left\langle R_{L}^{*}\right\rangle\right]$ is much lower than the total $\mathrm{rms}$ deviation $\left(\sigma_{2 \mathrm{rms}}\right)$ between the two quantities (cf. Table 4$)$. This is because the random part of the deviation cancels out when averaging over the entire dataset. In addition, if $\left[\left\langle R_{L_{0}}\right\rangle\right]$ instead of $\left[R_{L}^{*}\right]$ was used for comparison with $\left[\left\langle R_{L}\right\rangle\right]$, the results would be nearly identical since $\left[\left\langle R_{L}^{*}\right\rangle\right] \approx\left[\left\langle R_{L_{0}}\right\rangle\right]$ according to $(9)$. Thus, at large scale, bias due to beam-averaging identifies with bias due to intrinsic NUBF effects.

Results of the intrinsic NUBF effects study by Nakamura (1991), though performed for different conditions, exhibit bulk features comparable to those shown here: they produce positive bias in the $Z-R$ method and negative bias in attenuation algorithms except for a special case of the standard DF approach; all biases increase with the averaging spatial scale.

Analytical study of NUBF for SF algorithms (Amayenc et al. 1993) showed that the two main competing biasing effects were a bias $B_{1}$, due to the "concavity of the exponential PIA function" in cross-range averaging, which produces underestimation of the rain rate, increasing with the PIA, and a bias $B_{2}$, due to the "nonlinearity of $Z=\alpha k^{\beta}$ relations" used in the algorithms processing, which produces underestimation or overestimation of the rain rate, increasing with $\beta$. The observed features for $\mathrm{SF}$ algorithms are in bulk agreement with these predictions. At X band, bias $B_{2}$ likely compensates for, or dominates, bias $B_{1}$ owing to low PIA values and produces a final positive low bias. At $K_{a}$ band, bias $B_{1}$ dominates bias $B_{2}$ owing to much larger 
TABLE 4. Correlation coefficient $\rho_{1}\left(\rho_{2}\right)$ and relative rms deviation $\sigma_{1 \mathrm{rms}}\left(\sigma_{2 \mathrm{rms}}\right)$ between $\left\langle R_{L}\right\rangle$ and $\left\langle R_{L_{L}}\right\rangle\left(\left\langle R_{L}\right\rangle\right.$ and $\left.\left\langle R_{L}^{*}\right\rangle\right)$ for the rangeprofiling attenuation algorithms $k Z S_{\mathrm{X}}, k Z S_{\mathrm{K}}$, and $\mathrm{DF}$, respectively, and for different simulated cross-range resolution $L$. Here $R_{L}$ is the simulated beam-averaged rain rate; $R_{L}^{*}$ is an estimate of the true mean rain rate (averaged over $L$ ). The genuine resolution of the radar beam is $L_{0}=1 \mathrm{~km}$. The angle brackets stand for a height average of the involved quantity over a $2.5-\mathrm{km}$ depth above the surface. Results refer to the entire dataset (observations 2000-3600).

\begin{tabular}{clccc}
\hline \hline & & \multicolumn{3}{c}{ Cross-range resolution } \\
\cline { 3 - 5 } Algorithm & Parameter & $L=2 \mathrm{~km}$ & $L=3 \mathrm{~km}$ & $L=4 \mathrm{~km}$ \\
\hline \multirow{2}{*}{$k Z S_{\mathrm{X}}$} & $\rho_{1}$ & 0.981 & 0.976 & 0.968 \\
& $\sigma_{\text {Irms }}(\%)$ & 13 & 14 & 16 \\
& $\rho_{2}$ & 0.957 & 0.920 & 0.882 \\
& $\sigma_{2 \mathrm{rms}}(\%)$ & 21 & 28 & 33 \\
$k Z S_{\mathrm{K}}$ & $\rho_{1}$ & 0.972 & 0.956 & 0.947 \\
& $\sigma_{\text {1rms }}(\%)$ & 18 & 22 & 25 \\
& $\rho_{2}$ & 0.947 & 0.931 & 0.916 \\
& $\sigma_{2 \text { rms }}(\%)$ & 20 & 25 & 28 \\
$\mathrm{DF}$ & $\rho_{1}$ & 0.935 & 0.888 & 0.886 \\
& $\sigma_{\text {Irms }}(\%)$ & 30 & 34 & 36 \\
& $\rho_{2}$ & 0.909 & 0.866 & 0.862 \\
& $\sigma_{2 \mathrm{rms}}(\%)$ & 34 & 38 & 40 \\
\hline
\end{tabular}

PIA and produces a final negative bias. Results for the DF algorithm do not point out any superiority over the SF counterparts. This would not necessarily be the case for other frequency pairs.

The present results (Fig. 18b) also indicate that ranking of the algorithms with respect to their sensitivity to NUBF in decreasing merit order would yield first $Z R_{K}$, then $Z R_{\mathrm{X}}$, and finally the "attenuation" algorithms $k Z S_{\mathrm{X}}, k Z S_{\mathrm{K}}$, and DF, in that order. This is actually a puzzling result since it somewhat weakens the advantage of attenuation algorithms over $Z-R$ methods and of the DF approach over the SF counterparts to perform corrections of various scaling errors (cf. MA94, and section 4). The experimental data likely include such types of error and low NUBF effects at $L_{0}$ scale. Algorithms performances must be evaluated by comparing sensitivity to all causes of error among which possible impact of NUBF does not necessarily dominate. Attenuation algorithms, though possibly more biased by NUBF, can still be preferred to $Z-R$ methods that are almost unbiased by NUBF but ignore correction of path attenuation.

\section{Final discussion and conclusions}

Dual-frequency airborne radar data were used to study the performances of several SF algorithms and a DF algorithm aimed at profiling the rainfall rate versus range from a downward-looking spaceborne radar, as described in Part I (MA94). Such data mimic spaceborne radar measurements, including realistic error sources, such as an offset in the radar calibration, DSD variability, and $\sigma^{0}$ fluctuations. Most conditions, how- ever, are attached to the characteristics of the experiment: 10 and $35 \mathrm{GHz}$ and near-nadir beam pointing in the present case.

Rain retrievals were performed for stratiform and convective rain cases. In absence of "external" measurements to validate the rain estimates, we primarily checked the self-consistency of the results. The SF algorithms produce more or less scattered results depending on their own sensitivities to uncorrected scaling errors. Improvement of the SF approach stability by using surface echo measurements as a constraint on the total PIA ( $k Z C$ and $k Z S$ algorithms) was confirmed. The $k Z S$ algorithm, integrating from the surface, pro-
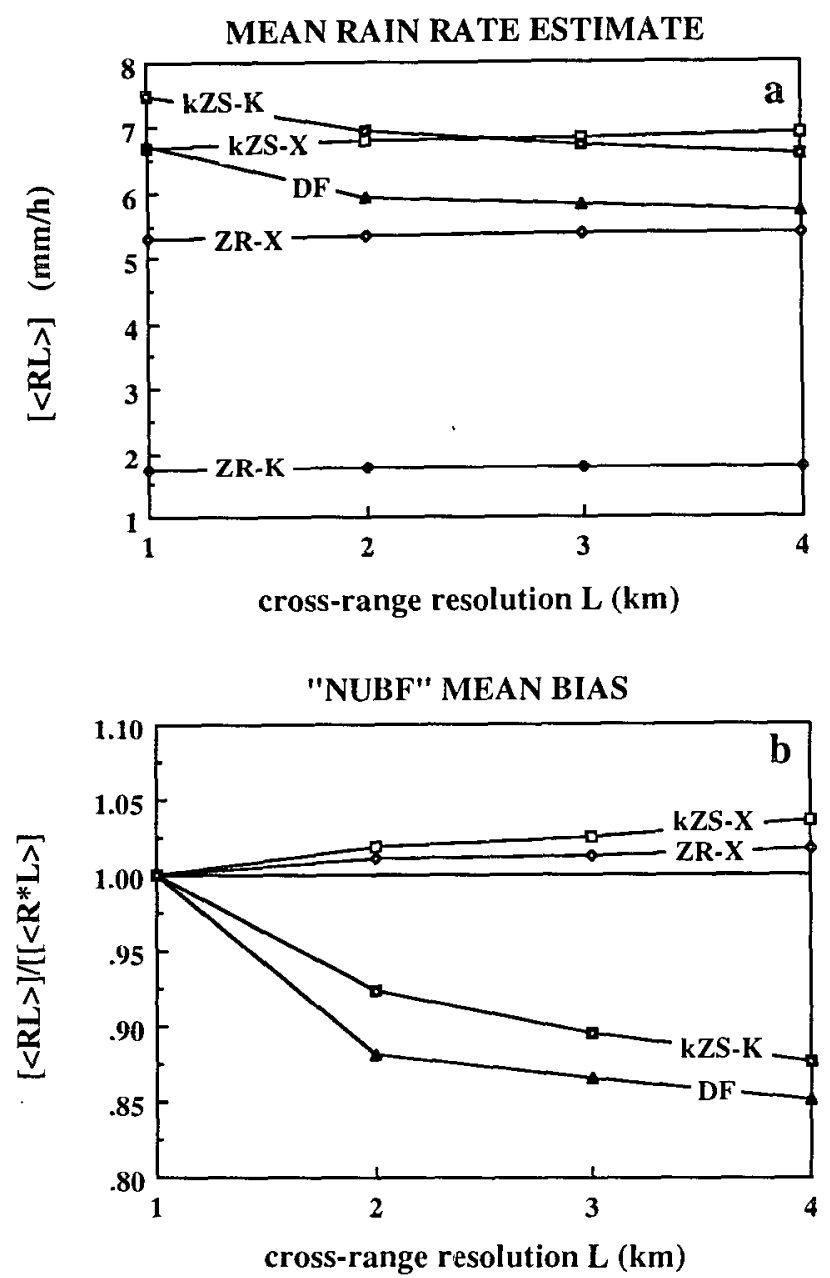

FIG. 18. Mean values over the entire dataset (observations 20003600 ), denoted by the square brackets of (a) $\left[\left\langle R_{L}\right\rangle\right]$ and (b) the ratio $\left[\left\langle R_{L}\right\rangle\right]\left[\left\langle R_{L}^{*}\right\rangle\right]^{-1}$, as function of the simulated cross-range resolution $L$. Results are shown for the range-profiling attenuation algorithms $k Z S_{\mathrm{X}}, k Z S_{\mathrm{K}}$, and DF, respectively. Results for $Z R_{\mathrm{X}}$, and $Z R_{\mathrm{K}}$ algorithms are also plotted for comparison. Here $R_{L}$ is the simulated beam-averaged rain rate; $R_{L}^{*}$ is an estimate of the true mean rain rate (averaged over $L$ ). Term $L_{0}=1 \mathrm{~km}$ is the genuine resolution of the radar beam. The angle brackets stand for the height average of the involved quantity over a $2.5-\mathrm{km}$ depth above the surface. 
duces generally the more reliable results, that is, the most similar at $\mathrm{X}$ band and $\mathrm{K}_{\mathrm{a}}$ band within the detectable rain rate range, and fairly converges toward $Z-R$ estimate for low PIA. The $k Z C$ algorithm works correctly for large PIA but produces scattered results at low PIA. Conversely, the $k Z$ algorithm, processing rain echoes only, works correctly at low PIA $(<3 \mathrm{~dB})$ but often collapses for large PIA $(>3 \mathrm{~dB})$. The $k Z N$ algorithm yields coherent results but may be penalized in the presence of significant height gradient of the rain rate near the surface.

The correlation between the attenuation coefficients at both frequencies, which forms the theoretical basis of the DF algorithm, was experimentally verified. Results from the DF algorithm are thought to be more reliable than the SF counterparts since they are globally corrected for scaling errors. Besides, the radar calibration correction derived from the DF algorithm results may be used to improve results of the $Z-R$ method, at least for low PIA. Results of rain profiling from $k Z S$ and DF algorithms allowed us to reconstruct coherent 2D raincell structures in a part of the observed rainy system.

The frequencies involved in the airborne radar data call for some comments. Use of radar data at X band and $\mathrm{K}_{\mathrm{a}}$ band has some drawbacks for the application of $\mathrm{SF}$ algorithms. At $\mathrm{X}$ band, low attenuation (for $R<5$ $\mathrm{mm} \mathrm{h}^{-1}$ ) may be masked by the variability in the $\sigma^{0}$ estimate. At $\mathrm{K}_{\mathrm{a}}$ band, large attenuation (for $R>20$ $\mathrm{mm} \mathrm{h}^{-1}$ ) can prevent detection of signal deep in the rain before the surface range. The DF approach, however, benefits by large differential attenuation effects. Since algorithm performances are mainly driven by the PIA, results at $K_{u}$ band and $K$ band, more usually planned for future spaceborne radars, may be inferred from relevant frequency-dependent $k-R$ relations. The mean rain-rate threshold $R_{T}$ above which "attenuation" algorithms should relay the $Z-R$ method to avoid negative bias in rain-rate estimates were found near 1 $\mathrm{mm} \mathrm{h}^{-1}$ at $35 \mathrm{GHz}$ and $12 \mathrm{~mm} \mathrm{~h}^{-1}$ at $10 \mathrm{GHz}$, respectively, for a $2.5-\mathrm{km}$ rain depth. This threshold would be $R_{T} \approx 1.5 \mathrm{~mm} \mathrm{~h}^{-1}$ at $24 \mathrm{GHz}$ and $\approx 5 \mathrm{~mm} \mathrm{~h}^{-1}$ at 14 $\mathrm{GHz}$, respectively. For constant rain rate, all thresholds correspond to a PIA $\approx 1 \mathrm{~dB}$, which is a lower limit for a reliable application of "attenuation" algorithms using surface echo measurements. Future work will involve tests of algorithms using data gathered over the western Pacific Ocean during TOGA COARE (Webster and Lukas 1992) with the Airborne Rain-Mapping Radar (ARMAR) at $\mathrm{K}_{\mathrm{u}}$ band (Durden et al. 1994) on board the DC8 aircraft of NASA.

The general features of the aforementioned results are in good agreement with previous works by other researchers who used similar datasets to test algorithms dedicated to rain profiling (Meneghini and Nakamura 1990; Kozu et al. 1991; Meneghini et al. 1992; Iguchi and Meneghini 1994). Though most numerical values are related to specific conditions of the experiment, the main conclusions applicable to other cases, also supported by results of Part I, are the superiority of "attenuation-compensating" over $Z-R$ methods to retrieve spatial structures or path averages of the rain rate, the primary control of the algorithm performances by the PIA amount in the merit-demerit balance, the benefit of using surface echo as a constraint to stabilize the SF algorithms over a large dynamic range, and the potential improvement brought by the DF approach over the SF counterparts to better correct scaling errors. Also, there is a promising way, initiated by Iguchi and Meneghini (1994), to investigate "hybrid" algorithms exploiting the well-identified complementary advantages of the various SF approaches.

The airborne radar measurements obtained with high cross-range resolution $\left(L_{0} \approx 1 \mathrm{~km}\right)$ were also used to simulate $2 \mathrm{D}$ beam-averaging effects and intrinsic NUBF effects for larger cross-range resolutions $L=2$, 3 , and $4 \mathrm{~km}$ in the alongtrack direction. Approximate computations of the relevant quantities were elaborated to point out both effects. Beam-averaging produces smoothing of small-scale rain-rate structures and bulk lowering of the rain rate as expected consequences of degrading the cross-range resolution. Intrinsic NUBF effects are characterized by deviations between the derived beam-averaged rain rate and the "true mean rain rate" over the beamwidth. They produce low positive bias, slowly increasing with $L$, for $k Z S_{\mathrm{X}}$ and more important negative bias, increasing with $L$, for $k Z S_{\mathrm{K}}$ and the DF algorithm. Impact of NUBF effects on mean rain-rate estimates at large scale $(\approx 100 \mathrm{~km})$, derived from attenuation algorithms, remains tolerable: the bias culminates near $-15 \%$ to $+2 \%$ for $L=4 \mathrm{~km}$, depending on the involved algorithm. The main feature, in overall agreement with results of Nakamura (1991), is that NUBF effects produce overestimation of the rain rate in $Z-R$ methods and underestimation of the rain rate in attenuation methods as soon as PIA becomes significant. The magnitude of NUBF effects, however, is governed by the measurement geometry and the attenuation rate. Thus, the obtained numerical results are inherently attached to the conditions of the experiment. They would not necessarily be the same for other conditions referring to frequencies, beam incidences, and storm structures.

It should finally be emphasized that an objective ranking of the bulk merits of the various algorithms has to appreciate their own sensitivities to all types of scaling errors possibly present in the measurements, not only to the NUBF.

Acknowledgments. This study received financial support from the Centre National d'Etudes Spatiales (France), and Comité PATOM de l'Institut National des Sciences de l'Univers (France). Part of the study also received financial support from the European Space Agency under Grant ESTEC 10204/92/NL/ $\mathrm{NB}(\mathrm{SC})$. We wish to thank Dr. R. Meneghini from 
NASA/GSFC for providing us the airborne radar dataset.

\section{APPENDIX}

\section{A Useful Expression to Estimate the PIA in the $\left(r, r_{s}\right)$ Range Interval above the Surface}

For the SF approach, a general expression of the twoway PIA factor $A\left(r, r_{s}\right)$ in a limited range interval ( $r$, $r_{s}$ ) above the surface, assuming detectable rain signal down to the surface range $r_{s}$, may be derived from MA94 [ see (17), with $r_{d}=r_{s}$ ], according to

$$
A\left(r, r_{s}\right)=\left[1+\left(\frac{y_{g}}{x_{g}}\right) S\left(r, r_{s}\right)\right]^{-\beta},
$$

where $S\left(r_{1}, r_{2}\right)$, given by

$$
S\left(r_{1}, r_{2}\right)=\int_{r_{1}}^{r_{2}} Z_{a m}^{1 / \beta}(s) d s,
$$

involves apparent reflectivity measurements $\left(Z_{a m}\right)$ within rain, and $\beta$ is a coefficient of the $Z=\alpha k^{\beta}$ relation. The expressions for $x_{g}$ and for $y_{g}=\left(1-x_{g}\right) /$ $S\left(r_{0}, r_{s}\right)$ depend on the involved algorithm (index $g$ ).

For $k Z S$ algorithm $(g=3), x_{3}$ derived from MA94 [see (24), with $r_{d}=r_{s}$ ] is given by

$$
x_{3}=\frac{\left(A_{t m} \alpha \delta A_{t} \delta C\right)^{1 / \beta}}{\left(A_{t m} \alpha \delta A_{t} \delta C\right)^{1 / \beta}+\gamma S\left(r_{0}, r_{s}\right)},
$$

where $\alpha$ is a coefficient of the $Z=\alpha k^{\beta}$ relation, $A_{t m}$ is the total PIA measured from surface echo attenuation below rain, $S\left(r_{0}, r_{s}\right)$ is defined by (A2), and $\gamma=0.46$ / $\beta$. The terms $\delta C$ and $\delta A_{t}$ represent possible uncorrected scaling errors in the radar calibration constant $C$ for rain echoes and in the $A_{t m}$ estimate, respectively. They are defined as multiplying factors of the assumed value.

Combining (A1), (A2), and (A3) yields the following expression for the two-way PIA factor $A\left(r, r_{s}\right)$ :

$$
\begin{aligned}
& A\left(r, r_{s}\right)=\left[1+\gamma \frac{S\left(r, r_{s}\right)}{\left(\alpha A_{t m} \delta A_{t} \delta C\right)^{1 / \beta}}\right]^{-\beta} \\
& \quad=\left[1+\left(\frac{0.46}{\beta}\right) \int_{r}^{r_{s}}\left(\frac{Z_{a m}(s)}{\alpha A_{t m} \delta A_{t} \delta C}\right)^{1 / \beta} d s\right]^{-\beta} .
\end{aligned}
$$

The $A\left(r, r_{s}\right)$ estimate, always positive, is obtained by integration between the surface range $r_{s}$ and any range $r$. Thus, like the $k(r)$ profile, it is independent of the storm structure at ranges less than $r$. It remains, however, sensitive to errors $\delta A_{t}$ that may arise from uncertainty in the $\sigma^{0}$ estimate due to effects of surface wind or drop impact over ocean, for example, and to uncertainty in the $\alpha$ coefficient due to DSD variability. The dependence on the $\beta$ parameter, which appears essentially as a norm in (A4), is almost negligible.
Sensitivity of $A\left(r, r_{s}\right)$ to an error $\delta C$ in the radar calibration referring to $Z_{a n}$ measurements in (A4), deserves further comment. The total PIA factor $A_{t m}$ is derived from the ratio of surface echo powers measured outside rain as a "reference" and below rain at different times and/or locations. This involves two possible additional errors in the radar calibration $C_{s}$ for surface echo measurements: $\delta C_{s 0}$ outside rain and $\delta C_{s}$ below rain. Since the radar returns from rain and the surface below rain are obtained "simultaneously" for the same path, $\delta C$ and $\delta C_{s}$ cancel out in the $Z_{a m} / A_{t m}$ ratio of (A4). Thus, $A\left(r, r_{s}\right)$ may be considered as insensitive to error in the radar calibration below rain but sensitive to a calibration error $\delta C_{s 0}$ in surface echo measurements outside rain only. Term $\delta C_{s 0}$, in turn, can be considered a part of the error in $A_{t m}$, thereby included in the bulk error $\delta A_{t}$. The same reasoning also applies to the $k(r)$ profile estimated from the $k Z S$ algorithm.

For a practical computation of $A\left(r, r_{s}\right)$ using (4), $Z_{a m}\left(A_{t m}\right)$ may be expressed as a function of returned power $P(r)$ from rain $\left[P_{s}\left(r_{s}\right)\right.$ from the surface], and error terms may be neglected by taking $\delta A_{t}=\delta C=1$, which yields finally

$$
\begin{aligned}
A\left(r, r_{s}\right)=[1 & +\left(\frac{0.46}{\beta}\right) \\
& \left.\times \int_{r}^{r_{s}}\left(\frac{P(r) r^{2} C_{s} \sigma^{0}}{P_{s}\left(r_{s}\right) r_{s}^{2} C \alpha}\right)^{1 / \beta} d r\right]^{-\beta},
\end{aligned}
$$

where $\sigma^{0}$ is the "reference" value of the surface backscattering coefficient.

\section{REFERENCES}

Amayenc, P., and M. Marzoug, 1992: A survey of algorithms studies developped at CRPE for range profiling of rain rate from a spaceborne radar. Proc. Int. Workshop on the Processing and Utilization of the Rainfall Data Measured from Space, Tokyo, Japan, Communications Research Laboratory, 289-294.

_ _, _- , and J. Testud, 1993: Analysis of cross-beam resolution effects in rainfall rate profile retrieval from a spaceborne radar. IEEE Trans. Geosci. Remote Sens., 31, 417-425.

Atlas, D., and C. W. Ulbrich, 1977: Path- and area-integrated rainfall measurement by microwave attenuation in the $1-3-\mathrm{cm}$ band. $J$. Appl. Meteor., 16, 1322-133.

Durden, S. L., E. Im, F. K. Li, W. Ricketts, A. Tanner, and W. Wilson, 1994: ARMAR: An Airborne Rain-Mapping Radar. J. Atmos. Oceanic Technol., 11, 727-737.

Fujita, M., K. Okamoto, M. Masuko, S. Yoshikado, and K. Nakamura, 1985: Inference of rain rate profile and path-integrated rain rate by an airborne microwave rain scatterometer. Radio Sci., 20, 631-642.

Goldhirsh, J., and E. J. Walsh, 1982: Rain measurements from space using a modified Seasat-type radar altimeter. IEEE Trans. Antenna Propag., 30, 726-732.

Hitschfeld, W., and J. Bordan, 1954: Errors inherent in the radar measurement of rainfall at attenuating wavelengths. J. Meteor., $11,58-67$.

Iguchi, T., and R. Meneghini, 1994: Intercomparison of single frequency methods for retrieving a vertical rain profile from airborne or spaceborne radar data. J. Atmos. Oceanic Technol, 11, $1507-1516$ 
Kozu, T., 1991: Estimation of raindrop size distribution from spaceborne measurement. Ph.D. thesis, Kyoto University, $196 \mathrm{pp}$.

-, K. Nakamura, R. Meneghini, and W. Boncyk, 1991: Dual parameter rainfall measurement from space: A test result from an aircraft experiment. IEEE Trans. Geosci. Remote Sens., 29, $690-703$.

Marshall, J. S., and W. M. K. Palmer, 1948: The distribution of raindrop with size. J. Meteor., 5, 165-166.

Marzoug, M., and P. Amayenc, 1991a: Experimental tests of an improved single frequency algorithm for rain rate profiling using airborne radar data. Proc. 25th Int. Conf. on Radar Meteorology, Paris, France, Amer. Meteor. Soc., 396-399.

- , and $-1991 \mathrm{~b}$ : Improved range profiling algorithm of rainfall rate from a spaceborne radar with path-integrated attenuation constraint. IEEE Trans. Geosci. Remote Sens. (Special issue IGARSS'90), GE-29, 584-592.

__ and _- 1992: A new class of dual-frequency algorithms for rain rate profiling from, a spaceborne radar: Principle and tests. Proc. IGARSS'92, Houston, TX, 1376-1379.

- , and _- 1993: Algorithms for range profiling of rain rate from a spaceborne radar: Overall validation using airborne radar data. Proc. 26th Conf. on Radar Meteorology, Norman, OK, Amer. Meteor. Soc., 690-693.

rithms for rain rate profiling from a spaceborne radar. Part I: Principle and tests from numerical simulations. J. Atmos. Oceanic Technol., 11, 1480-1506.

Meneghini, R., and T. Kozu, 1990: Spaceborne Weather Radar. Artech House, 199 pp.
_- and K. Nakamura, 1990: Range profiling of the rain rate by an airborne weather radar. Remote Sens. Environ., 31, 193-209.

- - J. A. Jones, and L. H. Gesell, 1987: Analysis of a dual-wavelength surface reference radar technique. IEEE Trans. Geosci. Remote Sents., 21, 34-43.

—, K. Nakamura, C. W. Ulbrich, and D. Atlas, 1989: Experimental tests of methods for the measurement of rainfall rate using an airborne dual-wavelength radar. J. Atmos. Oceanic Technol., 6, $637-651$.

_- T. Kozu, H. Kumagai, and W. C. Boncyck, 1992: A study of rain estimation methods from space using dual-wavelength radar measurements at near-nadir incidence over ocean. J. Atmos. Oceanic Technol., 9, 364-382.

Nakamura, K., 1991: Biases of rain retrieval algorithms for spaceborne radar caused by nonuniformity of rain. J. Atmos. Oceanic Technol., 8, 363-373.

Okamoto, K., T. Ojima, S. Yoshikado, H. Masuko, H. Inomata, and N. Fugono, 1982: System design and examination of spaceborne microwave rain scatterometer. Acta Astronaut., 9, 713-721.

Simpson, J., R. F. Adler, and G. R. North, 1988: A proposed Tropical Rainfall Measuring Mission (TRMM). Bull. Amer. Meteor. Soc., 69, 278-295.

Testud, J., P. Amayenc, and M. Marzoug, 1992: Rainfall rate retrieval from a spaceborne radar: Comparison between single-frequency, dual-frequency, and dual-beam techniques. J. Atmos. Oceanic Technol., 9, 599-623.

Webster, P. J., and R. Lukas, 1992: TOGA-COARE: The Coupled Ocean-Atmosphere Response Experiment. Bull. Amer. Meteor. Soc., 73, 1377-1416. 